\title{
Ranging Energy Optimization for Robust Sensor Positioning Based on Semidefinite Programming
}

\author{
Tao Wang, Geert Leus, Senior Member, IEEE, and Li Huang, Member, IEEE
}

\begin{abstract}
Sensor positioning is an important task of location-aware wireless sensor networks. In most sensor positioning systems, sensors and beacons need to emit ranging signals to each other. Sensor ranging energy should be low to prolong system lifetime, but sufficiently high to fulfill prescribed accuracy requirements. This motivates us to investigate ranging energy optimization problems. We address ranging energy optimization for an unsynchronized positioning system, which features robust sensor positioning (RSP) in the sense that a specific accuracy requirement is fulfilled within a prescribed service area. We assume a line-of-sight (LOS) channel exists between the sensor and each beacon. The positioning is implemented by time-of-arrival (TOA) based two-way ranging between a sensor and beacons, followed by a location estimation at a central processing unit. To establish a dependency between positioning accuracy and ranging energy, we assume the adopted TOA and location estimators are unbiased and attain the associated Cramér-Rao bound. The accuracy requirement has the same form as the one defined by the Federal Communication Commission (FCC), and we present two constraints with linear-matrix-inequality form for the RSP. Ranging energy optimization problems, as well as a practical algorithm based on semidefinite programming are proposed. The effectiveness of the algorithm is illustrated by numerical experiments.
\end{abstract}

Index Terms-Cramér-Rao bound, localization, semidefinite programming, wireless sensor networks.

\section{INTRODUCTION}

W IRELESS sensor networks (WSNs) enable a rich variety of promising applications, and therefore have attracted intensive research interest lately [1]. Typical WSNs consist of untethered sensors randomly deployed to collect application-specific measurements, as well as a few fusion centers for in-network data processing. For most WSNs, sensor positions have to be estimated first, because they are often indispensable to annotate sensed data. For instance, temperature data produced by an environment-monitoring WSN are

Manuscript received December 16, 2008; accepted June 18, 2009. First published July 21, 2009; current version published November 18, 2009. The associate editor coordinating the review of this manuscript and approving it for publication was Dr. Brian M. Sadler. Part of this paper was presented at International Conference on Acoustics, Speech and Signal Processing (ICASSP), Taipei, Taiwan, April 19-24, 2009.

T. Wang was with the Faculty of Electrical Engineering, Mathematics and Computer Science, Delft University of Technology, 2628 CD Delft, The Netherlands. He is also with the Stichting IMEC Nederland, Holst Centre, Eindhoven, The Netherlands (e-mail: t.wang@tudelft.nl).

G. Leus is with the Faculty of Electrical Engineering, Mathematics and Computer Science, Delft University of Technology, 2628 CD Delft, The Netherlands (e-mail: g.j.t.leus@tudelft.nl).

L. Huang is with the Stichting IMEC Nederland, Holst Centre, Eindhoven, The Netherlands (e-mail: Li.Huang@imec-nl.nl).

Color versions of one or more of the figures in this paper are available online at http://ieeexplore.ieee.org.

Digital Object Identifier 10.1109/TSP.2009.2028211 useless unless we know where they are sensed. Furthermore, some WSN applications, e.g., target tracking, require the sensor positions to be known a priori [2]. Especially for large-scale WSNs, the sensor positions are required for self-organization, such as naming [3], routing [4], as well as ciphering [5], just to name a few. Therefore, sensor positioning has been a research topic of particular interest over the past few years.

To support location-aware WSNs, sensor positions must be estimated reliably with prescribed accuracy requirements fulfilled. Perhaps the most well-known requirement is the one defined by the Federal Communication Commission (FCC) [6]. More specifically, it requires the location estimation error to have a length smaller than $R_{e}$ with probability higher than $P_{e}$, where both $R_{e}$ and $P_{e}$ have a prescribed value. Although this requirement has been introduced to regulate the localization of mobile users, it can be considered to prescribe an accuracy requirement for sensor positioning as well.

In practice, it is costly to equip each sensor with a global positioning system (GPS). Instead, a few beacons, which are sensors or fusion centers with known positions, are encompassed in most WSNs for locating the rest of the sensors in two steps. In the first step, beacons and sensors are scheduled to emit ranging signals to each other, and some signal parameters related to sensor positions are measured. Possible parameters include time-of-arrival (TOA), time-difference-of-arrival (TDOA), received-signal-strength (RSS), as well as angle-of-arrival (AOA) [7], [8]. In the second step, these measurements are transformed into distance or bearing information, from which the sensor positions are estimated by a specific algorithm with the use of the beacons' positions. In practice, all the above position-dependent parameters carry information about the sensor position, and an optimal localization algorithm should make use of all information related to the sensor position. However, the complexity may be too high especially when the localization task is partly carried out by the sensor. As a result, practical two-step algorithms usually estimate one or several dominant position-dependent parameters in the first step, and then estimate the sensor position in the second step. This incurs a loss of position-related information, and therefore practical algorithms have suboptimal performance in general. However, they are preferred for WSNs where the reduction of implementation complexity is a big concern.

To locate sensors with high accuracy, TOA measurements are preferred, since TDOA measurements require multiple synchronized beacons, RSS measurements need an accurate pathloss model, and antenna arrays are necessary for AOA measurements [7]. Thanks to the superior penetration and resolution capability of ultrawideband (UWB), TOA-based ranging using 
UWB pulses has been regarded as the most promising candidate for WSNs. Especially for unsynchronized WSNs, TOAbased two-way ranging (TWR) is proposed and supported by the IEEE 802.15.4a standard [9]. Recently, a variety of work on TOA-based ranging has been reported in [10]-[17]. Particularly, the associated CRBs have been derived to benchmark the performance of a particular algorithm [7], [18]-[21].

As for the second step, two types of positioning algorithms have been developed. There is a rich literature on noncollaborative positioning algorithms, which consider only the aforementioned ranging between the sensor and the beacons [22]-[31]. Recently, collaborative positioning algorithms, which employ additional inter-sensor ranging for performance improvement, have been developed [32]-[37]. The associated CRBs have been derived in [8], [38]-[40], and [41]-[44] to benchmark the performance of a noncollaborative and a collaborative algorithm, respectively.

For the above two-step algorithms, positioning accuracy improves if the ranging energy of sensors and beacons is enhanced. In real scenarios, a beacon might have a reliable power supply and its ranging energy can be easily increased, but the ranging energy of an untethered sensor must be reduced in order to prolong system lifetime. Therefore, the positioning accuracy is actually dominated by the sensor ranging energy, which should be small but sufficiently high to fulfill prescribed accuracy requirements. This motivates us to investigate the following ranging energy optimization problem: how to allocate the ranging energy to sensors and beacons, so that the sensor ranging energy is minimized and specific accuracy requirements are fulfilled as well?

We will address this problem for an unsynchronized robust sensor positioning (RSP) system, which consists of power-supplied beacons connected to a central processing unit (CPU), as well as sensors randomly deployed within a prescribed service area. We assume a line-of-sight (LOS) channel exists between the sensor and each beacon. The positioning is implemented by TOA-based TWR between a sensor and the beacons, followed by a location estimation at the CPU with a noncollaborative algorithm. In particular, this system features RSP, in the sense that a specific accuracy requirement is fulfilled within a prescribed service area. To reduce the implementation complexity, the ranging energy of both the sensor and the beacons is fixed and determined during the system design phase. In order to establish a mathematical dependency between the positioning accuracy and the ranging energy, we assume the adopted TOA estimator achieves its CRB with an unbiased Gaussian distribution, and the positioning CRB is achieved by an unbiased location estimator. The motivation behind these assumptions is twofold. One is that although our assumptions seem too optimistic in practice, the associated positioning CRB has a very attractive mathematical structure which lends the proposed problems to be efficiently solved by semidefinite programming (SDP). The other is that the optimal ranging energy allocation for a real scenario is actually lower bounded by the result produced by SDP for the optimistically assumed scenario. Therefore, our assumptions lead to useful optimization results, which can not only be found efficiently by SDP, but also provide a sense of how much energy should be allocated at least.

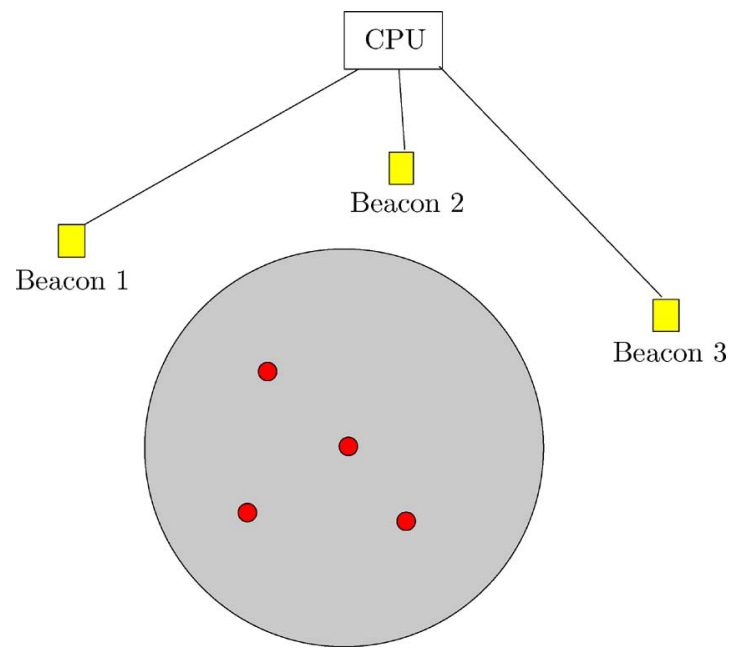

Fig. 1. An exemplary sensor positioning system, where the circular area and the small circles represent the service area and sensors, respectively.

The rest of this paper is organized as follows. In the next section, we will derive the performance of the considered TOA and location estimators. Next, Section III will present the RSP constraints when the location estimate is Gaussian distributed, and when such statistical knowledge is unavailable. After that, we will propose ranging energy optimization problems and a practical algorithm based on SDP in Section IV. In Section V, we will illustrate the effectiveness of the proposed algorithm by numerical experiments. Finally, we will wrap up this paper by some conclusions in Section VI.

\section{System Setur AND Performance ANAlysis}

In this section, we will first describe a two-dimensional (2D) RSP system. Next, a TOA-based TWR procedure is introduced and its performance is derived. Finally, the performance of the adopted positioning algorithm, which is actually the positioning $\mathrm{CRB}$, is derived.

\section{A. System Setup}

We study a 2D RSP system with $M$ beacons deployed and connected to a CPU through wired or radio links $(M \geq 3)$. Beacon $m$ is at a known coordinate $\mathbf{p}_{m}=\left[x_{m}, y_{m}\right]^{T}, m=$ $1, \cdots, M$, and we will consider locating a sensor at an unknown coordinate $\mathbf{u}=\left[x_{\mathrm{s}}, y_{\mathrm{s}}\right]^{T}$ within a prescribed service area $S$. The distance between the sensor and beacon $m$ is denoted as $d_{m, \mathbf{u}}=\left\|\mathbf{u}-\mathbf{p}_{m}\right\|_{2}$, where $\|\cdot\|_{2}$ represents the $l_{2}$-norm operator. In addition, the clocks of the sensor and the beacons are unsynchronized but run at the same pace. We assume that the two-sided power spectral density (PSD) of the additive white Gaussian noise (AWGN) at the sensor and the beacons is $N_{\mathrm{S}} / 2$ and $N_{\mathrm{b}} / 2$, respectively. For illustration purposes, Fig. 1 shows an exemplary system with a circular service area and a few sensors.

We assume a LOS channel exists between the sensor and beacon $m$, which incurs a propagation delay $d_{m, \mathbf{u}} / c$ and attenuation $a_{m}$. Here $c$ represents the signal propagation speed and $a_{m}^{2}=\alpha\left(d_{m, \mathbf{u}}\right)^{-\beta}$, where $\alpha$ and $\beta$ refer to the path gain at $1 \mathrm{~m}$ and the path-loss coefficient, respectively. We assume $\alpha$ and $\beta$ are both known by system designers. 
During the ranging phase, the sensor and beacon $m$ are scheduled to broadcast respectively the ranging signals $s_{\mathrm{s}}(t)$ and $s_{m}(t)$. In practice, a ranging signal can be the preamble part of a communication signal between the sensor and beacon $m$ [9]. Note that these signals are not necessarily UWB in general. Finally, we assume the channel remains unchanged during the ranging phase, and both the signal TOA and $a_{m}$ are regarded as unknown deterministic parameters by the adopted TOA and location estimators.

It has been shown in [7], [20], and [45] that the CRB of TOA estimation depends on the root-mean-square (RMS) angular frequency of the adopted ranging signals. We assume all beacon ranging signals have the same RMS angular frequency. To facilitate the following derivations, we define the RMS angular frequency of $s_{\mathrm{s}}(t)$ and $s_{m}(t)$ respectively as

$$
\begin{aligned}
& \omega_{\mathrm{s}}=\sqrt{\frac{\int_{-\infty}^{+\infty}\left|2 \pi f S_{\mathrm{s}}(f)\right|^{2} d f}{\int_{-\infty}^{+\infty}\left|S_{\mathrm{s}}(f)\right|^{2} d f}} \\
& \omega_{\mathrm{b}}=\sqrt{\frac{\int_{-\infty}^{+\infty}\left|2 \pi f S_{m}(f)\right|^{2} d f}{\int_{-\infty}^{+\infty}\left|S_{m}(f)\right|^{2} d f}}
\end{aligned}
$$

where $S_{\mathrm{s}}(f)$ and $S_{m}(f)$ represent the spectrum of $s_{\mathrm{s}}(t)$ and $s_{m}(t)$, respectively.

\section{B. TOA-Based TWR and Performance}

TOA-based TWR proceeds as follows [9]. First, the CPU schedules the beacons to broadcast ranging signals sequentially, so that they are separated when arriving at the sensor. Let's say beacon $m$ broadcasts $s_{m}(t)$ of energy $E_{m}$ at time $T_{m}$. At the sensor, the LOS signal's TOA $T_{m \rightarrow \mathrm{s}}$ is estimated and denoted as $\hat{T}_{m \rightarrow \mathrm{s}}$. After the sensor has generated all the TOA estimates, it broadcasts $s_{\mathrm{s}}(t)$ of energy $E_{\mathrm{s}}$ at time $T_{\mathrm{s}}$ to the beacons. At beacon $m$, the LOS signal's TOA $T_{\mathrm{s} \rightarrow m}$ is estimated and denoted as $\hat{T}_{\mathrm{S} \rightarrow m}$. We assume hardware calibration is perfectly accomplished by the sensor and beacon $m$, so that $T_{\mathrm{s}}$ and $T_{m}$ are precisely known by the sensor and beacon $m$, respectively. It is important to note that $\hat{T}_{m \rightarrow \mathrm{s}}$ and $T_{\mathrm{s}}$ are recorded by the sensor with its internal clock, while $\hat{T}_{\mathrm{s} \rightarrow m}$ and $T_{m}$ are recorded by beacon $m$ with its internal clock. Finally, all the processing delays $\left\{T_{\mathrm{s}}-\hat{T}_{m \rightarrow \mathrm{s}}\right\}_{m=1}^{M}$ produced by the sensor are first transmitted through data packets to the beacons, and then sent to the CPU. Meanwhile, the total delay $\hat{T}_{\mathrm{s} \rightarrow m}-T_{m}$ generated by beacon $m$ is also transmitted to the CPU, which evaluates $\hat{d}_{m, \mathbf{u}}$ as

$$
\hat{d}_{m, \mathbf{u}}=\frac{c}{2}\left[\left(\hat{T}_{\mathrm{s} \rightarrow m}-T_{m}\right)-\left(T_{\mathrm{s}}-\hat{T}_{m \rightarrow \mathrm{s}}\right)\right] .
$$

Ideally, if $T_{m \rightarrow \mathrm{s}}$ and $T_{\mathrm{s} \rightarrow m}$ are precisely estimated, $\hat{d}_{m, \mathbf{u}}$ is equal to $d_{m, \mathbf{u}}$. However, the estimation performance is degraded by the AWGN. It is well known that the variance of any unbiased estimator is lower-bounded by the CRB. We assume each TOA estimator for the TWR achieves the CRB with an unbiased Gaussian distribution. In fact, this can be asymptotically accomplished by the maximum-likelihood (ML) estimator [10], [18]. The CRB has been derived in [21] when the distribution of the propagation delay and $a_{m}$ is known a priori, and in [7],
[18]-[20] for scenarios similar to the one considered here. For self-consistency purposes, we put a brief derivation of the CRB in Appendix A, where we show that $\hat{T}_{m \rightarrow \mathrm{s}}$ is Gaussian distributed as $\mathcal{N}\left(T_{m \rightarrow \mathrm{s}}, \sigma_{m \rightarrow \mathrm{s}}^{2}\right)$, and $\sigma_{m \rightarrow \mathrm{s}}^{2}$ is expressed by

$$
\sigma_{m \rightarrow \mathrm{s}}^{2}=\frac{N_{\mathrm{s}} / 2}{a_{m}^{2} \omega_{\mathrm{b}}^{2} E_{m}} .
$$

Similarly, $\hat{T}_{\mathrm{S} \rightarrow m}$ has the Gaussian distribution $\mathcal{N}\left(T_{\mathrm{s} \rightarrow m}, \sigma_{\mathrm{s} \rightarrow m}^{2}\right)$, where $\sigma_{\mathrm{s} \rightarrow m}^{2}$ is evaluated as

$$
\sigma_{\mathrm{s} \rightarrow m}^{2}=\frac{N_{\mathrm{b}} / 2}{a_{m}^{2} \omega_{\mathrm{s}}^{2} E_{\mathrm{s}}} .
$$

We can see that $\hat{T}_{m \rightarrow \mathrm{s}}$ and $\hat{T}_{\mathrm{s} \rightarrow m}$ are independent since they are generated from independent signals. Therefore, $\hat{d}_{m, \mathbf{u}}$ is distributed as $\mathcal{N}\left(d_{m, \mathbf{u}}, \kappa_{m}^{2}\right)$, where $\kappa_{m}^{2}$ is given by

$$
\begin{aligned}
\kappa_{m}^{2} & =\frac{c^{2}}{4}\left(\sigma_{m \rightarrow \mathrm{s}}^{2}+\sigma_{\mathrm{s} \rightarrow m}^{2}\right) \\
& =\rho a_{m}^{-2}\left(E_{\mathrm{s}}^{-1}+\gamma E_{m}^{-1}\right)
\end{aligned}
$$

with $\rho=c^{2} N_{\mathrm{b}} /\left(8 \omega_{\mathrm{s}}^{2}\right)$ and $\gamma=N_{\mathrm{s}} \omega_{\mathrm{s}}^{2} /\left(N_{\mathrm{b}} \omega_{\mathrm{b}}^{2}\right)$. In fact, $\gamma$ represents the TOA estimation accuracy of the sensor relative to that of beacon $m$ when $E_{\mathrm{s}}=E_{m}(\gamma<1$ means that the TOA estimation of the sensor is more accurate).

\section{Performance of the Positioning Algorithm}

After TWR, a set of independent measurements $\left\{\hat{d}_{m, \mathbf{u}}\right\}_{m=1}^{M}$ is available at the CPU for estimating $\mathbf{u}$. For any unbiased location estimator, the CRB $\mathbf{C}_{\mathbf{u}}$ sets a lower-bound to its covariance. The CRBs for similar scenarios have been derived in [8] and [38]-[40]; however, they did not consider the presence of $\left\{a_{m}\right\}_{m=1}^{M}$ as nuisance parameters, and thus the results reported there can't be used here. In Appendix B, we show $\mathbf{C}_{\mathbf{u}}$ can be evaluated according to

$$
\mathbf{C}_{\mathbf{u}}^{-1}=\mathbf{F}(\mathbf{x}, \mathbf{u})=\sum_{m=1}^{M} x_{m} \mathbf{F}_{m}(\mathbf{u})
$$

where $\mathbf{F}_{m}(\mathbf{u})=\alpha \rho^{-1}\left(d_{m, \mathbf{u}}\right)^{-\beta-2}\left(\mathbf{u}-\mathbf{p}_{m}\right)\left(\mathbf{u}-\mathbf{p}_{m}\right)^{T}$, and $\mathbf{x}=\left[x_{1}, \cdots, x_{M}\right]^{T}$. Here, $x_{m}=\left(E_{\mathrm{s}}^{-1}+\gamma E_{m}^{-1}\right)^{-1}$ can be regarded as the effective energy that combines the joint effect of $E_{\mathrm{s}}$ and $E_{m}$ on $\mathbf{C}_{\mathbf{u}}$. Note that $x_{m} \leq \min \left\{E_{\mathrm{s}}, E_{m} / \gamma\right\}$, and $x_{m}$ is an increasing function of both $E_{\mathrm{s}}$ and $E_{m}$.

One may assume that the location estimate $\hat{\mathbf{u}}$ follows the Gaussian distribution $\mathcal{N}\left(\mathbf{u}, \mathbf{C}_{\mathbf{u}}\right)$. Such an assumption is attractive because the Gaussian distribution has salient mathematical properties and therefore can ease theoretical analysis. For instance, this assumption was adopted for localization performance analysis in [8] and [46]. In addition, the Gaussian-distribution assumption can be justified since the CRB can be achieved with an unbiased Gaussian distribution by the ML estimator, provided that we have a large set of independent and identically distributed (i.i.d.) ranging measurements [45]. Although such a large set is usually unavailable in practice, it has been shown in [25] that the estimator based on constrained weighted least-squares could approach the CRB if the ranging accuracy is sufficiently high, and the distribution of the location estimate resembles an unbiased Gaussian distribution. 
On the other hand, although the location estimators based on approximate ML [24], [27], and multidimensional scaling (MDS) [31] can also achieve the CRB, they do not necessarily follow a Gaussian distribution.

Based on the above considerations, we assume $\hat{\mathbf{u}}$ is unbiased and its covariance attains $\mathbf{C}_{\mathbf{u}}$, while leaving the assumption about the distribution of $\hat{\mathbf{u}}$ open. In the next section, we will derive constraints for RSP when $\hat{\mathbf{u}}$ is Gaussian distributed, as well as when the knowledge about the distribution of $\hat{\mathbf{u}}$ is unavailable.

\section{Comments}

It is important to examine more closely the assumptions we made. First, we assumed the TOA estimation CRB can be achieved. However, it was shown in [47], [48] that this $\mathrm{CRB}$ is in nature a local bound and not tight for scenarios with low signal-to-noise ratio (SNR). Alternatively, one may consider the improved Ziv-Zakai bound (ZZB) as a better metric. Second, we assumed a LOS channel exists between the sensor and each beacon, which may be too optimistic for real scenarios. Especially in indoor or dense urban environments, the LOS path may not exist and even if it exists, there may exist strong multipath interference on the LOS signal. Third, we assumed each ranging estimate is only degraded by the TOA estimation error and thus Gaussian distributed. In practice, it may be further degraded by the random behavior of queuing the ranging signals at the sensor and beacons.

Our assumptions are motivated by the fact that if we take other bounds, such as the ZZB, or more realistic assumptions, finding the optimal ranging energy allocation for the proposed problems is generally very difficult. Although our assumptions seem too optimistic in practice, the associated CRB computed from (8) has a very attractive mathematical structure which lends the proposed problems to be efficiently solved by SDP. Furthermore, the optimal ranging energy allocation for a real scenario is actually lower bounded by the result produced by SDP for the optimistically assumed scenario. Therefore, our assumptions lead to useful optimization results, which can not only be found efficiently by SDP, but also provide a sense of how much energy should be allocated at least.

Note that it is very attractive to investigate the gap between the optimal energy allocation under our assumptions and that for real scenarios, when more knowledge is available about the statistics of the channels, the signal queuing, and the implemented TOA and position estimation algorithms. Intuitively, this gap should be translated from the localization performance loss due to the impairments present in a real scenario but ignored by our assumptions. This topic is part of our future work.

\section{CONSTRAINTS FOR RSP}

To support location-awareness reliably, we require the sensor to be located with sufficiently high accuracy. To this end, we impose an RSP requirement on the considered system using the FCC definition. More specifically, we require that for every $\mathbf{u}$ within $S$, the location estimation error $\mathbf{e}=\hat{\mathbf{u}}-\mathbf{u}$ falls into the origin-centered circle of radius $R_{e}$ with probability higher than $P_{e}$, i.e., $\forall \mathbf{u} \in S, \operatorname{Pr}\left(\|\mathbf{e}\|_{2} \leq R_{e}\right) \geq P_{e}$. Note that $R_{e}$ and $P_{e}$ can be prescribed according to the accuracy level expected by location-dependent functions. We can see that a higher accuracy level is expected by reducing $R_{e}$ and increasing $P_{e}$.

In the following subsections, we will propose constraints for RSP when $\hat{\mathbf{u}}$ is Gaussian distributed, as well as when the knowledge about the distribution of $\hat{\mathbf{u}}$ is unavailable.

\section{A. Gaussian Distribution of the Location Estimate}

In this case, a sufficient condition to satisfy the accuracy requirement at $\mathbf{u}$ has been introduced in [8] as $\lambda_{\min }(\mathbf{x}, \mathbf{u}) \geq \lambda_{\mathrm{g}}$, where $\lambda_{\min }(\mathbf{x}, \mathbf{u})$ is the minimal eigenvalue of $\mathbf{F}(\mathbf{x}, \mathbf{u})$, and $\lambda_{\mathrm{g}}$ is the threshold translated from $P_{e}$ and $R_{e}$ according to

$$
\lambda_{\mathrm{g}}=\frac{2}{R_{e}^{2}} \ln \left(\frac{1}{1-P_{e}}\right) \text {. }
$$

This sufficient condition can be justified as follows. Since $\hat{\mathbf{u}}$ is distributed as $\mathcal{N}\left(\mathbf{u}, \mathbf{C}_{\mathbf{u}}\right)$, it was shown in [46] that $\mathbf{e}$ falls within an ellipse expressed by $\Phi_{e}=\left\{\mathbf{e}: \mathbf{e}^{T} \mathbf{F}(\mathbf{x}, \mathbf{u}) \mathbf{e} \leq-2 \ln (1-\right.$ $\left.\left.P_{e}\right)\right\}$ with probability $P_{e}$. This ellipse has a major principal axis of length $\sqrt{-2 \ln \left(1-P_{e}\right) / \lambda_{\min }(\mathbf{x}, \mathbf{u})}$. When $\lambda_{\min }(\mathbf{x}, \mathbf{u}) \geq$ $\lambda_{\mathrm{g}}$ is satisfied, $\Phi_{e}$ is contained within the circle $\Phi_{c}=\{\mathbf{e}$ : $\left.\|\mathbf{e}\|_{2} \leq R_{e}\right\}$, which means that $\operatorname{Pr}\left(\mathbf{e} \in \Phi_{c}\right) \geq \operatorname{Pr}\left(\mathbf{e} \in \Phi_{e}\right)=$ $P_{e}$. Therefore, $\lambda_{\min }(\mathbf{x}, \mathbf{u}) \geq \lambda_{\mathrm{g}}$ is sufficient to fulfill the accuracy requirement at $\mathbf{u}$.

Note that $\lambda_{\min }(\mathbf{x}, \mathbf{u}) \geq \lambda_{\mathrm{g}}$ is equivalent to the linear matrix inequality (LMI) $\sum_{m=1}^{M} x_{m} \mathbf{F}_{m}(\mathbf{u}) \succeq \lambda_{\mathrm{g}} \mathbf{I}$, where $\mathbf{I}$ is a $2 \times$ 2 identity matrix, and $\mathbf{A} \succeq \mathbf{B}$ means that $\mathbf{A}-\mathbf{B}$ is a positive semidefinite matrix. According to the convex optimization theory, the set of x's satisfying this LMI is convex [49]. With this constraint, convex optimization methods can be used to effectively solve the optimization problems proposed in Section IV.

To satisfy the RSP requirement, we require the considered system to fulfill this sufficient condition for every position within $S$, namely $\forall \mathbf{u} \in S, \lambda_{\min }(\mathbf{x}, \mathbf{u}) \geq \lambda_{\mathrm{g}}$.

\section{B. Unknown Distribution of the Location Estimate}

In this case, a sufficient condition to satisfy the accuracy requirement at $\mathbf{u}$ will be derived as $\lambda_{\min }(\mathbf{x}, \mathbf{u}) \geq \lambda_{\mathrm{r}}$. In the following, we will show that $\lambda_{\mathrm{r}}$ can be translated from $P_{e}$ and $R_{e}$ according to

$$
\lambda_{\mathrm{r}}=\frac{2}{R_{e}^{2}} \frac{1}{1-P_{e}} .
$$

This sufficient condition can be derived according to the Chebyshev's inequality, which is of the form $\operatorname{Pr}\left(\|\mathbf{e}\|_{2} \geq R_{e}\right) \leq \operatorname{Tr}\left(\mathbf{C}_{\mathbf{u}}\right) / R_{e}^{2}$ for the considered system [50]. Here, $\operatorname{Tr}\left(\mathbf{C}_{\mathbf{u}}\right)$ denotes the trace of $\mathbf{C}_{\mathbf{u}}$ and is equal to the mean square error of $\hat{\mathbf{u}}$. Note that this inequality holds for any particular distribution of $\hat{\mathbf{u}}$, and thus can be used to develop a sufficient condition when the knowledge about the distribution of $\hat{\mathbf{u}}$ is unavailable. As a result, $\operatorname{Pr}\left(\|\mathbf{e}\|_{2} \leq R_{e}\right) \geq 1-\operatorname{Tr}\left(\mathbf{C}_{\mathbf{u}}\right) / R_{e}^{2}$, and it is sufficient to satisfy the accuracy requirement if $1-\operatorname{Tr}\left(\mathbf{C}_{\mathbf{u}}\right) / R_{e}^{2} \geq P_{e}$, or equivalently $\operatorname{Tr}\left(\mathbf{C}_{\mathbf{u}}\right) \leq\left(1-P_{e}\right) R_{e}^{2}$. Referring to (8), each eigenvalue of $\mathbf{C}_{\mathbf{u}}^{-1}$ is greater than $\lambda_{\min }(\mathbf{x}, \mathbf{u})$, which means that each eigenvalue of $\mathbf{C}_{\mathbf{u}}$ is upper bounded by $\lambda_{\min }(\mathbf{x}, \mathbf{u})^{-1}$. As a result, $\operatorname{Tr}\left(\mathbf{C}_{\mathbf{u}}\right) \leq 2 \lambda_{\min }(\mathbf{x}, \mathbf{u})^{-1}$. Therefore, a stronger sufficient condition is $2 \lambda_{\min }(\mathbf{x}, \mathbf{u})^{-1} \leq\left(1-P_{e}\right) R_{e}^{2}$, or equivalently $\lambda_{\min }(\mathbf{x}, \mathbf{u}) \geq \lambda_{\mathrm{r}}$. 
Note that $\operatorname{Tr}\left(\mathbf{C}_{\mathbf{u}}\right) \leq\left(1-P_{e}\right) R_{e}^{2}$ is a sufficient condition as well. However, the associated set of feasible x's forms a nonconvex set in general, and this will make it difficult to solve the optimization problems proposed in Section IV. Therefore, we choose the stronger condition $\lambda_{\min }(\mathbf{x}, \mathbf{u}) \geq \lambda_{\mathrm{r}}$.

To satisfy the RSP requirement, we require the considered system to fulfill the proposed sufficient condition at every position within $S$, namely $\forall \mathbf{u} \in S, \lambda_{\min }(\mathbf{x}, \mathbf{u}) \geq \lambda_{\mathrm{r}}$.

\section{Comments}

The second RSP constraint is stronger than the first one, since $1 /\left(1-P_{e}\right)>\ln \left(1 /\left(1-P_{e}\right)\right)$ for any $P_{e} \in(0,1)$. This is because the first one is derived with extra knowledge about the error ellipse of a Gaussian distribution. With the second constraint, the RSP can be achieved no matter how $\hat{\mathbf{u}}$ is distributed. This means that using the second constraint guarantees the RSP even though the knowledge about the distribution of $\hat{\mathbf{u}}$ is unavailable.

To facilitate the discussion in the following sections, we formulate a general RSP constraint as: $\forall \mathbf{u} \in S, \lambda_{\min }(\mathbf{x}, \mathbf{u}) \geq \lambda$, where $\lambda=\lambda_{\mathrm{g}}$ if we know $\hat{\mathbf{u}}$ is Gaussian distributed, or $\lambda=\lambda_{\mathrm{r}}$ if we have no knowledge about the distribution of $\hat{\mathbf{u}}$.

\section{RANGING ENERGY OPTIMIZATION}

In this section, we will first formulate a few ranging energy optimization problems of interest under the RSP constraint proposed in Section III. Then, we will present a practical algorithm to solve them based on semidefinite programming (SDP). Note that $E_{\mathrm{s}},\left\{E_{m}\right\}_{m=1}^{M}, \mathbf{X}$ are all treated as optimization variables on which the equality constraints $x_{m}=\left(E_{\mathrm{s}}^{-1}+\gamma E_{m}^{-1}\right)^{-1}$, $m=1, \cdots, M$ are imposed.

\section{A. Sensor Ranging Energy Optimization Problem}

We first prove that $\lambda_{\min }(\mathbf{x}, \mathbf{u})$ is a nondecreasing function of $x_{m}, m=1, \cdots, M$. Suppose $x_{m}$ is increased by a positive value $\varepsilon_{m}$. As a consequence, $\mathbf{x}, \mathbf{F}(\mathbf{x}, \mathbf{u})$ and $\lambda_{\min }(\mathbf{x}, \mathbf{u})$ become $\mathbf{x}^{\prime}, \mathbf{F}\left(\mathbf{x}^{\prime}, \mathbf{u}\right)=\mathbf{F}(\mathbf{x}, \mathbf{u})+\varepsilon_{m} \mathbf{F}_{m}(\mathbf{u})$ and $\lambda_{\min }\left(\mathbf{x}^{\prime}, \mathbf{u}\right)$, respectively. Since $\varepsilon_{m} \mathbf{F}_{m}(\mathbf{u})$ is a positive semidefinite matrix, $\lambda_{\min }\left(\mathbf{x}^{\prime}, \mathbf{u}\right) \geq \lambda_{\min }(\mathbf{x}, \mathbf{u})$ according to Corollary 4.3.3 in [51], which justifies our claim. Since $\lambda_{\min }(\mathbf{x}, \mathbf{u})$ is nondecreasing with $x_{m}$, the RSP constraint can be fulfilled by increasing entries of $\mathbf{x}$, which in turn is accomplished by enhancing $E_{\mathrm{s}}$ and $E_{m}$.

It is important to note that $\|\mathbf{x}\|_{\infty} \leq E_{\mathrm{s}}$, where $\|\mathbf{x}\|_{\infty}=$ $\max _{m=1, \cdots, M}\left|x_{m}\right|$ denotes the $l_{\infty}$-norm of $\mathbf{x}$, and the equality holds when at least one $E_{m}$ increases to be infinity. To prolong system life time, $E_{\mathrm{s}}$ should be reduced as much as possible. However, in case $E_{\mathrm{s}}$ is reduced too much, every entry of $\mathbf{x}$ will be bounded by a too small $E_{\mathrm{s}}$ to fulfill the RSP constraint. This motivates us to find a threshold $E_{\text {th }}$ for $E_{\mathrm{s}}$, above which RSP becomes possible. Mathematically, $E_{\mathrm{th}}$ can be computed as the optimal objective value of the following optimization problem:

$$
\begin{aligned}
& \min _{E_{\mathrm{s}},\left\{E_{m}\right\}_{m=1}^{M}, \mathbf{x}} \quad E_{\mathrm{s}} \\
& \text { s.t. } \quad E_{\mathrm{s}} \geq 0 \\
& E_{m} \geq 0, \quad m=1, \cdots, M \\
& x_{m}=\left(E_{\mathrm{s}}^{-1}+\gamma E_{m}^{-1}\right)^{-1}, \quad m=1, \cdots, M \\
& \lambda_{\min }(\mathbf{x}, \mathbf{u}) \geq \lambda, \quad \forall \mathbf{u} \in S .
\end{aligned}
$$

We present the following theorem to compute $E_{\mathrm{th}}$.

Theorem 1: $E_{\text {th }}=\lambda / \min _{\mathbf{u} \in S}\left\{\lambda_{\min }(\mathbf{X}(\mathbf{u}))\right\}$, where $\lambda_{\min }(\mathbf{X}(\mathbf{u}))$ denotes the minimal eigenvalue of $\mathbf{X}(\mathbf{u})=$ $\sum_{m=1}^{M} \mathbf{F}_{m}(\mathbf{u})$

Proof: Let's consider the following optimization problem:

$$
\begin{aligned}
\min _{E_{\mathrm{s}}, \mathbf{x}} & E_{\mathrm{s}} \\
\text { s.t. } & x_{m}=E_{\mathrm{s}} \geq 0, m=1, \cdots, M \\
& \lambda_{\min }(\mathbf{x}, \mathbf{u}) \geq \lambda, \quad \forall \mathbf{u} \in S .
\end{aligned}
$$

Note that $E_{\mathrm{s}}$ and $\mathrm{x}$ are the only optimization variables in (12). Alternatively, (12) can be regarded as a simplification of (11) when every $E_{m}$ approaches infinity. With the equality constraint $x_{m}=E_{\mathrm{s}} \geq 0$, we can easily find that the inequality constraint in (12) reduces to $\lambda_{\min }(\mathbf{x}, \mathbf{u})=E_{\mathrm{s}} \lambda_{\min }(\mathbf{X}(\mathbf{u})) \geq \lambda, \forall \mathbf{u} \in S$. This means that each feasible $E_{\mathrm{s}}$ satisfies $E_{\mathrm{s}} \geq \max _{\mathbf{u} \in S}\left\{\lambda / \lambda_{\min }(\mathbf{X}(\mathbf{u}))\right\}=$ $\lambda / \min _{\mathbf{u} \in S}\left\{\lambda_{\min }(\mathbf{X}(\mathbf{u}))\right\}$. As a result, the optimal objective value is $E_{\mathrm{o}}=\lambda / \min _{\mathbf{u} \in S}\left\{\lambda_{\min }(\mathbf{X}(\mathbf{u}))\right\}$.

We can express $E_{\text {th }}$ and $E_{\mathrm{o}}$ alternatively as

$$
E_{\mathrm{th}}=\min _{E_{\mathrm{s}} \in \Gamma_{1}}\left\{E_{\mathrm{s}}\right\}, \quad E_{\mathrm{o}}=\min _{E_{\mathrm{s}} \in \Gamma_{2}}\left\{E_{\mathrm{s}}\right\}
$$

where $\Gamma_{1}$ and $\Gamma_{2}$ are the feasible sets of $E_{\mathrm{S}}$ for the problems (11) and (12), respectively.

Apparently, $E_{\mathrm{th}} \leq E_{\mathrm{o}}$ since $\Gamma_{2} \subseteq \Gamma_{1}$. Suppose now that $E_{\text {th }}<E_{\mathrm{O}}$. This means that there exists at least one particular $\mathbf{x}_{\mathrm{f}}$ that fulfills the constraints of (11) and $\left\|\mathbf{x}_{\mathrm{f}}\right\|_{\infty}=E_{\mathrm{th}}$. We can build a vector $\mathbf{x}_{\mathrm{th}}=\left[E_{\mathrm{th}}, \cdots, E_{\mathrm{th}}\right]^{T}$. Obviously, each entry of $\mathbf{x}_{\text {th }}$ is no smaller than that of $\mathbf{x}_{\mathbf{f}}$. Since $\lambda_{\min }(\mathbf{x}, \mathbf{u})$ is nondecreasing with $x_{m}, \lambda_{\min }\left(\mathbf{x}_{\mathrm{th}}, \mathbf{u}\right) \geq \lambda_{\min }\left(\mathbf{x}_{\mathrm{f}}, \mathbf{u}\right) \geq \lambda$, and therefore $\mathbf{x}_{\mathrm{th}}$ is feasible for (12). This means $E_{\mathrm{s}}=E_{\mathrm{th}}$ is a possible objective value of (12), but this value is smaller than $E_{\mathrm{o}}$, and thus the fact that $E_{\mathrm{o}}$ is the minimal objective value of (12) is violated. This contradicts the assumption $E_{\mathrm{th}}<E_{\mathrm{O}}$. Therefore, $E_{\text {th }}=E_{\text {o }}$.

We can see that $E_{\text {th }}$ is actually the minimal $l_{\infty}$-norm of any $\mathbf{x}$ fulfilling the RSP constraint. This implies that at least one $E_{m}$ has to be infinitely high, in case $E_{\mathrm{s}}$ is reduced to its lowest level $E_{\text {th }}$. It is interesting to observe that, if $\lambda$ is increased from $\lambda_{\mathrm{g}}$ to $\lambda_{\mathrm{r}}, E_{\mathrm{th}}$ has to be increased by $10 \log _{10}(\varsigma) \mathrm{dBJ}$, where $\varsigma=$ $\lambda_{\mathrm{r}} / \lambda_{\mathrm{g}}=-\left[\left(1-P_{e}\right) \ln \left(1-P_{e}\right)\right]^{-1}$. In effect, this increase can be regarded as the extra sensor energy required to compensate for the unavailable knowledge about the distribution of $\hat{\mathbf{u}}$ when $E_{m}$ ideally approaches infinity.

It is also interesting to study the impact of the path loss coefficient $\beta$ on $E_{\mathrm{th}}$. In order to facilitate the following discussion, let's denote $\eta=\min _{\mathbf{u} \in S}\left\{\lambda_{\min }(\mathbf{X}(\mathbf{u}))\right\}$ and, thus, $E_{\mathrm{th}} / \lambda=\eta^{-1}$. In fact, $\mathbf{X}(\mathbf{u})=\sum_{m=1}^{M} \mathbf{F}_{m}(\mathbf{u})=$ $\sum_{m=1}^{M}\left(d_{m, \mathbf{u}}\right)^{-\beta-2} \mathbf{Y}_{m}(\mathbf{u})$ where $\mathbf{Y}_{m}(\mathbf{u})=\alpha \rho^{-1}(\mathbf{u}-$ $\left.\mathbf{p}_{m}\right)\left(\mathbf{u}-\mathbf{p}_{m}\right)^{T}$. Suppose after $\beta$ increases, $\eta$ and $\mathbf{X}(\mathbf{u})$ change to $\eta^{\prime}$ and $\mathbf{X}^{\prime}(\mathbf{u})$, respectively. Since $\left(d_{m, \mathbf{u}}\right)^{-\beta-2}$ reduces and each $\mathbf{Y}_{m}(\mathbf{u})$ is positive semidefinite, $\lambda_{\min }\left(\mathbf{X}^{\prime}(\mathbf{u})\right) \leq$ $\lambda_{\min }(\mathbf{X}(\mathbf{u}))$ holds according to Corollary 4.3.3 in [51]. Suppose $\eta=\lambda_{\min }\left(\mathbf{X}\left(\mathbf{u}_{1}\right)\right)$, we have $\eta^{\prime}=\min _{\mathbf{u} \in S}\left\{\lambda_{\min }\left(\mathbf{X}^{\prime}(\mathbf{u})\right)\right\} \leq$ $\lambda_{\min }\left(\mathbf{X}^{\prime}\left(\mathbf{u}_{1}\right)\right) \leq \lambda_{\min }\left(\mathbf{X}\left(\mathbf{u}_{1}\right)\right)=\eta$. This means that $\eta$ is nonincreasing with $\beta$. Therefore, the threshold sensor energy 
will not reduce, if the signal energy decays faster with respect to the distance.

\section{B. Sensor and Beacon Ranging Energy Optimization Problems}

In real scenarios, we can not simply adopt the energy allocation $E_{\mathrm{s}}=E_{\mathrm{th}}, E_{m}=+\infty$ for at least one beacon, because $E_{m}$ is usually constrained by a prescribed value $E_{\mathrm{B}}$ due to implementation difficulties, e.g., short ranging duration or limited power due to power-amplifier nonlinearity. Note that the selected $E_{\mathrm{B}}$ must be no smaller than $\gamma E_{\mathrm{th}}$ in order to fulfill the RSP constraint. This is because $\|\mathbf{x}\|_{\infty} \leq E_{\mathrm{B}} / \gamma$ since $x_{m} \leq$ $\min \left\{E_{\mathrm{s}}, E_{m} / \gamma\right\}$ and $E_{m} \leq E_{\mathrm{B}}$ hold. Besides, $E_{\mathrm{th}} \leq\|\mathbf{x}\|_{\infty}$ must be satisfied in order to fulfill the RSP constraint. Therefore, $E_{\mathrm{B}}$ must be no smaller than $\gamma E_{\mathrm{th}}$ in order to fulfill the RSP constraint.

The optimization problem now is to find the minimal $E_{\mathrm{s}}$ and the associated $E_{m}$ which meet the RSP constraint. Mathematically, this problem can be cast into the following form:

$$
\begin{aligned}
& \min _{E_{\mathrm{s}},\left\{E_{m}\right\}_{m=1}^{M}, \mathbf{x}} \quad E_{\mathrm{s}} \\
& \text { s.t. } E_{\mathrm{s}} \geq 0 \\
& E_{\mathrm{B}} \geq E_{m} \geq 0, \quad m=1, \cdots, M \\
& x_{m}=\left(E_{\mathrm{s}}^{-1}+\gamma E_{m}^{-1}\right)^{-1}, \quad m=1, \cdots, M \\
& \lambda_{\min }(\mathbf{x}, \mathbf{u}) \geq \lambda, \quad \forall \mathbf{u} \in S .
\end{aligned}
$$

Let's denote the optimal solution to (14) by $E_{\mathrm{s}}^{*},\left\{E_{m}^{*}\right\}_{m=1}^{M}$ and $\mathrm{x}^{*}$. We present a general solution to the above problem with the following theorem.

Theorem 2: The general solution to (14) is

$$
\begin{aligned}
& E_{\mathrm{s}}^{*}=\left(E_{\mathrm{th}}^{-1}-\gamma E_{\mathrm{B}}^{-1}\right)^{-1} \\
& E_{m}^{*}=\gamma\left(x_{m}^{*-1}-E_{\mathrm{th}}^{-1}+\gamma E_{\mathrm{B}}^{-1}\right)^{-1}
\end{aligned}
$$

where $x_{m}^{*}$ is the $m$ th entry of $\mathbf{x}^{*}$, which belongs to the set $\Xi=$ $\left\{\mathbf{x}:\|\mathbf{x}\|_{\infty}=E_{\text {th }}\right.$ and $\left.\forall \mathbf{u} \in S, \lambda_{\min }(\mathbf{x}, \mathbf{u}) \geq \lambda\right\}$.

Proof: First of all, after some simple mathematical manipulations we can see that the $E_{\mathrm{s}}^{*}$ and $E_{m}^{*}$ given above satisfy the equality constraint in (14). Note that $E_{\mathrm{B}}$ must be no less than $\gamma E_{\mathrm{th}}$ in order to fulfill the RSP constraint, and thus $E_{\mathrm{s}}^{*}$ is nonnegative. Besides, since $\left\|\mathrm{x}^{*}\right\|_{\infty}=E_{\mathrm{th}}, x_{m}^{*} \leq E_{\mathrm{th}}$ and hence $E_{m}^{*}$ is also nonnegative. Therefore, $E_{\mathrm{s}}^{*}$ and $E_{m}^{*}$ belong to the feasible set of (14).

Second, $\left(E_{\mathrm{th}}^{-1}-\gamma E_{\mathrm{B}}^{-1}\right)^{-1}$ is the optimal value of $E_{\mathrm{S}}$, because once $E_{\mathrm{s}}$ becomes smaller than that value, the RSP constraint can not be satisfied since $\|\mathrm{x}\|_{\infty}<E_{\mathrm{th}}$. The reason for this is that for any $m=1, \cdots, M, x_{m}=\left(E_{\mathrm{s}}^{-1}+\gamma E_{m}^{-1}\right)^{-1}<$ $E_{\text {th }}$ when $E_{\mathrm{s}}<\left(E_{\mathrm{th}}^{-1}-\gamma E_{\mathrm{B}}^{-1}\right)^{-1}$ and $E_{m} \leq E_{\mathrm{B}}$, since $x_{m}$ is increasing with $E_{\mathrm{s}}$ and $E_{m}$.

Third, when $E_{\mathrm{s}}=E_{\mathrm{s}}^{*},\|\mathbf{x}\|_{\infty} \leq E_{\text {th }}$. To fulfill the RSP constraint, $\|\mathbf{x}\|_{\infty}$ must be equal to $E_{\text {th }}$ and thus $\mathbf{x}^{*}$ must belong to $\Xi$. Therefore, $E_{m}^{*}$ can be found by (16).

At least one solution to (14) exists and is given by

$$
E_{\mathrm{s}}^{*}=\left(E_{\mathrm{th}}^{-1}-\gamma E_{\mathrm{B}}^{-1}\right)^{-1}, \quad E_{m}^{*}=E_{\mathrm{B}} .
$$

In fact, this solution is constructed with $\mathrm{x}^{*}=\mathrm{x}_{\mathrm{th}}=$ $\left[E_{\mathrm{th}}, \cdots, E_{\mathrm{th}}\right]^{T}$ in $\Xi$. This solution is the worst one if $\Xi$ contains more than one element, since using any $\mathbf{x}^{*} \in \Xi$ other than $\mathbf{x}_{\mathrm{th}}$, another solution with less beacon energy usage can be constructed. For all those solutions, the optimal value of $E_{\mathrm{s}}$ remains fixed at $E_{\mathrm{s}}^{*}=\left(E_{\mathrm{th}}^{-1}-\gamma E_{\mathrm{B}}^{-1}\right)^{-1}$. Apparently, this value is greater than $E_{\mathrm{th}}$, and the gap $E_{\triangle}$ between $E_{\mathrm{s}}^{*}$ and $E_{\mathrm{th}}$ can be computed in $\mathrm{dBJ}$ as follows:

$$
E_{\triangle}=10 \log _{10} \frac{E_{\mathrm{s}}^{*}}{E_{\mathrm{th}}}=-10 \log _{10}\left(1-\frac{\gamma E_{\mathrm{th}}}{E_{\mathrm{B}}}\right) .
$$

We can see that $E_{\triangle}$ reduces as $E_{\mathrm{B}}$ increases, which means that $E_{\mathrm{s}}^{*}$ can approach $E_{\mathrm{th}}$ closer if $E_{\mathrm{B}}$ increases. In practice, (18) can be used to establish a tradeoff between $E_{\mathrm{s}}^{*}$ and $E_{\mathrm{B}}$, which is helpful to guide ranging hardware design of beacons and sensors. We can also see that for a fixed $E_{\mathrm{B}}, E_{\triangle}$ increases as $E_{\mathrm{th}}$ increases. This means that if $\lambda$ changes from $\lambda_{\mathrm{g}}$ to $\lambda_{\mathrm{r}}$, the gap between $E_{\mathrm{s}}^{*}$ and $E_{\mathrm{th}}$ becomes greater.

Instead of using the solution in (17), another solution with a more efficient use of beacon energy can be constructed by using in (16) the $\mathrm{x}^{*} \in \Xi$ obtained by solving the following problem:

$$
\begin{array}{ll}
\min _{\mathbf{x}} & \mathbf{w}^{T} \mathbf{x} \\
\text { s.t. } & E_{\text {th }} \geq x_{m} \geq 0, \quad m=1, \cdots, M \\
& \lambda_{\min }(\mathbf{x}, \mathbf{u}) \geq \lambda, \quad \forall \mathbf{u} \in S
\end{array}
$$

where $\mathbf{w}=\left[w_{1}, \cdots, w_{M}\right]^{T}$ is a weighting vector. We assume it is designed under the constraints that $w_{m} \in[0,1]$ and $\sum_{m=1}^{M} w_{m}=1$. Particularly, assigning a greater $w_{m}$ represents a stronger expectation to reduce $E_{m}$. One special case of interest is to set all entries of $\mathbf{w}$ to zero except for the $q$ th entry, i.e., $w_{q}=1$ and $w_{m}=0$ if $m \neq q$. Using the associated optimal $\mathrm{x}$ for (19), the constructed $E_{m}^{*}$ 's by (16) reduce the ranging energy of beacon $q$ to its minimal possible value with the RSP constraint satisfied.

In general, no closed-form solution exists for (19). Nevertheless, $\lambda_{\min }(\mathbf{x}, \mathbf{u}) \geq \lambda$ is equivalent to the LMI $\sum_{m=1}^{M} x_{m} \mathbf{F}_{m}(\mathbf{u}) \succeq \lambda \mathbf{I}$, and, thus, (19) actually belongs to the class of SDP problems, which can be solved numerically with convex optimization techniques [49].

\section{A Practical Algorithm}

To evaluate $E_{\text {th }}$ and solve (19), the main difficulty lies in the fact that $S$ is in general a continuous area. A practical algorithm is realized by replacing $S$ with a discrete grid set $G=\left\{\mathbf{g}_{n}\right\}_{n=1}^{N}$, where $\mathbf{g}_{n}$ is the $n$th grid point within $S$. Then, we can evaluate $E_{\text {th }}$ and solve (19) using numerical convex optimization software, such as Sedumi [52].

Suppose that $G$ is generated by sampling $S$ uniformly in both vertical and horizontal directions with a spacing $\triangle_{0}$. Ideally, $\triangle_{0}$ should approach zero as close as possible. But if $\triangle_{0}$ is too small, the optimization problems will go beyond the processing ability of the optimization software, since too many LMI constraints are produced. This motivates us to choose a reasonably small value for $\triangle_{0}$. To this end, let's consider $\Omega_{S}$ and $\Omega_{G}$, which denote the sets of $\mathbf{x}$ fulfilling the RSP constraint over $S$ and $G$, respectively. Obviously, $\Omega_{S} \subseteq \Omega_{G}$, and $\triangle_{0}$ should be sufficiently small such that $\Omega_{G} \approx \Omega_{S}$.

To find a principle for choosing $\triangle_{0}$, consider a square cell $C_{\triangle}$ of lateral length $\triangle$ centered at a grid point $\mathrm{g}_{c} \in S$. Such a 


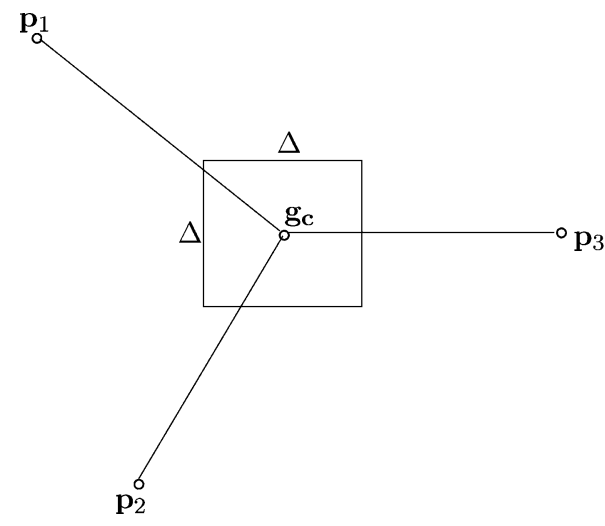

Fig. 2. An exemplary square cell $C_{\triangle}$ and its position relative to three beacons.

cell is shown in Fig. 2 for illustration purposes. Within this cell, $\lambda_{\min }(\mathbf{x}, \mathbf{u})$ has the following property.

Theorem 3: $\lambda_{\min }(\mathbf{x}, \mathbf{u})$ is approximately a concave function of $\mathbf{u} \in C_{\triangle}$, provided that $\triangle \ll d_{\min }\left(\mathbf{g}_{c}\right)$, where $d_{\min }\left(\mathrm{g}_{c}\right)=$ $\min _{m \in\{1, \cdots, M\}}\left\{d_{m, \mathbf{g}_{c}}\right\}$ refers to the distance of $\mathbf{g}_{c}$ to the closest beacon. In addition, if $\lambda_{\min }\left(\mathbf{x}, \mathbf{g}_{j}\right) \geq \lambda$ holds at four corner points $\left\{\mathbf{g}_{j}\right\}_{j=1}^{4}, \lambda_{\min }(\mathbf{x}, \mathbf{u}) \geq \lambda$ for every $\mathbf{u} \in S$.

Proof: When $\triangle \ll d_{\min }\left(\mathbf{g}_{c}\right),\left\|\mathbf{u}-\mathbf{g}_{c}\right\|_{2} \ll\left\|\mathbf{g}_{c}-\mathbf{p}_{m}\right\|_{2}$, and thus $\left\|\mathbf{u}-\mathbf{p}_{m}\right\|_{2} \approx\left\|\mathbf{g}_{c}-\mathbf{p}_{m}\right\|_{2}$ and $\alpha \rho^{-1}\left(d_{m, \mathbf{u}}\right)^{-\beta-2} \approx$ $\alpha \rho^{-1}\left(d_{m, \mathbf{g}_{c}}\right)^{-\beta-2}=\mu_{m}$ hold for every $\mathbf{u} \in C_{\triangle}$.

Therefore, $\lambda_{\min }(\mathbf{x}, \mathbf{u})$ can be approximated as follows:

$$
\begin{aligned}
& \lambda_{\min }(\mathbf{x}, \mathbf{u}) \\
& \quad=\min _{\mathbf{v}} \sum_{m=1}^{M} x_{m} \mathbf{v}^{T} \mathbf{F}_{m}(\mathbf{u}) \mathbf{v} \\
& \quad \approx \min _{\mathbf{v}} \sum_{m=1}^{M} x_{m} \mu_{m}\left|\mathbf{v}^{T}\left(\mathbf{u}-\mathbf{g}_{c}\right)+\mathbf{v}^{T}\left(\mathbf{g}_{c}-\mathbf{p}_{m}\right)\right|^{2} \\
& \quad \approx \min _{\mathbf{v}} \sum_{m=1}^{M} x_{m} \mu_{m}\left(\mathbf{v}^{T} \mathbf{Z}_{m} \mathbf{v}+2 \mathbf{v}^{T}\left(\mathbf{g}_{c}-\mathbf{p}_{m}\right) \mathbf{v}^{T}\left(\mathbf{u}-\mathbf{g}_{c}\right)\right)
\end{aligned}
$$

where $\|\mathbf{v}\|_{2}=1$ and $\mathbf{Z}_{m}=\left(\mathbf{g}_{c}-\mathbf{p}_{m}\right)\left(\mathbf{g}_{c}-\mathbf{p}_{m}\right)^{T}$.

Obviously, the function inside the last min operator is an affine function of $\mathbf{u}$ parameterized by $\mathbf{v}$ and $\mathbf{x}$, so $\lambda_{\min }(\mathbf{x}, \mathbf{u})$ is approximately a pointwise minimum of this function over $\mathbf{v}$. Therefore, $\forall \mathbf{x}, \lambda_{\min }(\mathbf{x}, \mathbf{u})$ is approximately a concave function of $\mathbf{u} \in C_{\triangle}$ [49].

In fact, $C_{\triangle}$ is the convex hull formed by its corner points $\left\{\mathbf{g}_{j}\right\}_{j=1}^{4}$. This means that $\forall \mathbf{u} \in C_{\triangle}$, there exists a set $\left\{\theta_{j}\right\}_{j=1}^{4}$ where $\theta_{j} \in[0,1]$ and $\sum_{j=1}^{4} \theta_{j}=1$, such that $\mathbf{u}=\sum_{j=1}^{4} \theta_{j} \mathbf{g}_{j}$ [49]. If $\forall j, \lambda_{\min }\left(\mathbf{x}, \mathbf{g}_{j}\right) \geq \lambda$ holds, $\lambda_{\min }(\mathbf{x}, \mathbf{u}) \geq \lambda$ is satisfied within $C_{\triangle}$ because of the concavity of $\lambda_{\min }(\mathbf{x}, \mathbf{u})$ over $\mathbf{u} \in C_{\triangle}$

$$
\lambda_{\min }(\mathbf{x}, \mathbf{u}) \geq \sum_{j=1}^{4} \theta_{j} \lambda_{\min }\left(\mathbf{x}, \mathbf{g}_{j}\right) \geq \sum_{j=1}^{4} \theta_{j} \lambda=\lambda .
$$

Based on Theorem 3, if the RSP constraint is fulfilled at all corner points, the RSP constraint will be satisfied over the whole $C_{\triangle}$. As a result, $\triangle_{0}$ should be chosen according to $\triangle_{0} \ll$

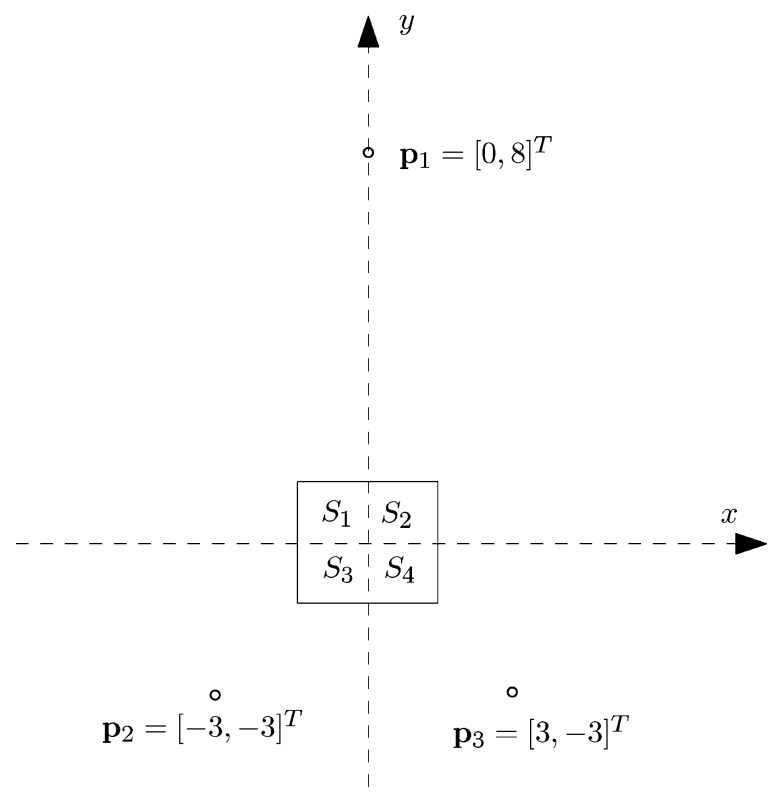

Fig. 3. System setup and subregions.

$\min _{\mathbf{u} \in S} d_{\min }(\mathbf{u})$. Under this condition, using any $\mathbf{x}$ fulfilling the RSP constraint over $G$, the RSP constraint is satisfied over $S$ as well, because the RSP constraint is satisfied within each cell formed by four adjacent points in $G$, and those cells cover $S$.

To use the above sampling condition, we assume that all beacons lie outside $S$. It is important to note that, if one beacon is very close to $S, \min _{\mathbf{u} \in S} d_{\min }(\mathbf{u})$ will be very small. In order to reduce the size of $G$, we can sample $S$ nonuniformly with the following method. First, divide $S$ into $Q$ subregions $\left\{S_{q}\right\}_{q=1}^{Q}$. Then we sample each region $S_{q}$ uniformly with a spacing $\triangle_{q} \ll$ $\min _{\mathbf{u} \in S_{q}} d_{\min }(\mathbf{u})$ to produce a discrete set $G_{q}$. Finally, $G$ is produced by combining all $G_{q}$ 's as: $G=\bigcup_{q=1}^{Q} G_{q}$. Based on a similar analysis as that for uniform sampling, we can then justify $\Omega_{G} \approx \Omega_{S}$. Apparently, $\triangle_{q} \geq \triangle_{0}$, so $G$ has a size no greater than that produced by uniform sampling.

\section{NUMERICAL EXPERIMENTS}

For illustration purposes, numerical experiments have been conducted using the system setup shown in Fig. 3. Suppose $S$ is a square area centered at $(0,0)$ with lateral length $2 \mathrm{~m}$. There are three beacons located at $\mathbf{p}_{1}=[0,8]^{T}, \mathbf{p}_{2}=[-3,-3]^{T}$, and $\mathbf{p}_{3}=[3,-3]^{T}$, respectively. Note that the unit for all those values is meter. The system parameters are set as: $\alpha=1$, $c=3 \times 10^{8} \mathrm{~m} / \mathrm{s}, N_{\mathrm{s}} / 2=N_{\mathrm{b}} / 2=0 \mathrm{dBW} / \mathrm{Hz}$, and $\omega_{\mathrm{s}} / 2 \pi=$ $\omega_{\mathrm{b}} / 2 \pi=8 \mathrm{GHz}$. We will set $\beta=2$ in the following experiments unless otherwise stated. All computations are performed with Matlab v7.1 and Sedumi v1.1 on a laptop equipped with an AMD Turion CPU of speed $2.2 \mathrm{GHz}$ and a memory of $2 \mathrm{G}$ Bytes.

For the RSP constraint, we prescribe $R_{e}$ to be fixed at $6 \mathrm{~cm}$. As we expect a better accuracy level by increasing $P_{e}$ from 0.7 to $0.95, \lambda_{\mathrm{g}}$ increases from 28.3 to $32.2 \mathrm{~dB}$ as shown in Fig. 4. When $\lambda$ changes from $\lambda_{\mathrm{g}}$ to $\lambda_{\mathrm{r}}$, the increase of $E_{\mathrm{th}}$ in $\mathrm{dBJ}$ is equal to $10 \log _{10}\left(\lambda_{\mathrm{r}} / \lambda_{\mathrm{g}}\right)$, which is shown in Fig. 4 as well. In effect, this increase is the extra sensor energy required to compensate for the unavailable knowledge about the distribution of 

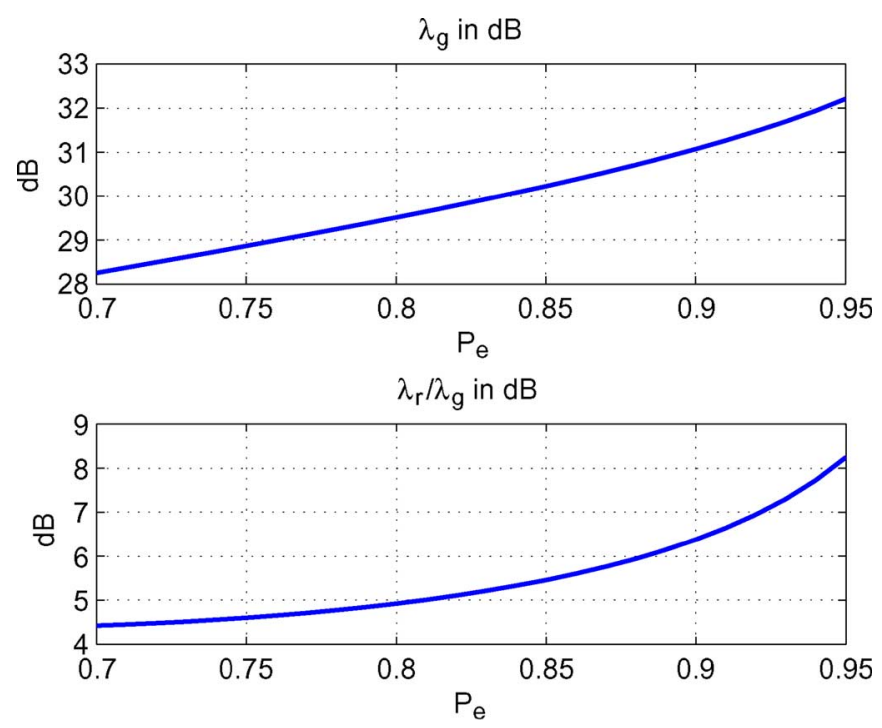

Fig. 4. The computed results for $\lambda_{\mathrm{g}}$ and $\lambda_{\mathrm{r}} / \lambda_{\mathrm{g}}$ when $R_{e}=6 \mathrm{~cm}$.
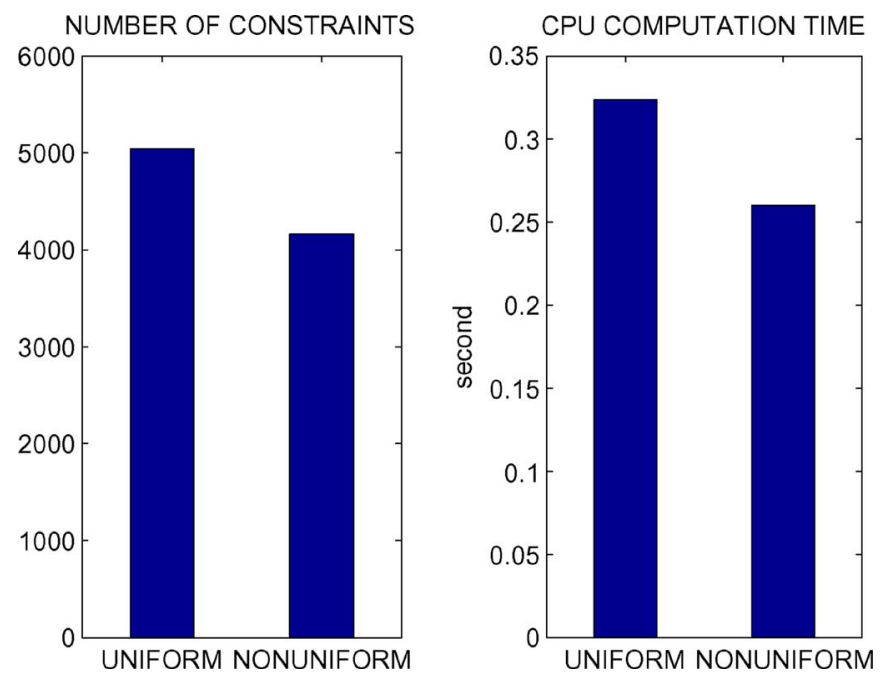

Fig. 5. Number of constraints and computation time.

$\hat{\mathbf{u}}$, when $E_{\mathrm{s}}$ attains its lowest level $E_{\mathrm{th}}$ and at least one $E_{m}$ approaches infinity. It is shown that this extra energy is between 4.4 and $8.2 \mathrm{dBJ}$, and a higher $P_{e}$ demands a higher extra energy. In the following experiments, we choose $P_{e}=0.85$ which corresponds to an extra energy of about 5.6 dBJ.

To evaluate $E_{\mathrm{th}}, S$ should be replaced with a discrete set $G$. Both uniform and nonuniform sampling are performed to produce $G$. For the uniform sampling, we use a spacing $\triangle_{0}=\delta \cdot d_{0}$ where $d_{0}=\min _{\mathbf{u} \in S} d_{\min }(\mathbf{u})$. For the nonuniform sampling, we divide $S$ into four subregions $\left\{S_{q}\right\}_{q=1}^{4}$ by $x=0$ and $y=0$ as shown in Fig. 3, and $S_{q}$ is sampled uniformly with a spacing $\triangle_{q}=\delta \cdot d_{q}$ where $d_{q}=\min _{\mathbf{u} \in S_{q}} d_{\min }(\mathbf{u})$. Here, $\delta$ is a parameter for tuning the sampling spacing. During the evaluation, we find that when $\delta \leq 1 \%$ the computed $E_{\text {th }}$ remains essentially unchanged as -4.32 and $1.14 \mathrm{dBJ}$ for $\lambda=\lambda_{\mathrm{g}}$ and $\lambda=\lambda_{\mathrm{r}}$, respectively. This implies that $\Omega_{G}$ is quite close to $\Omega_{S}$ when $\delta$ is below $1 \%$. When the nonuniform sampling is used instead of

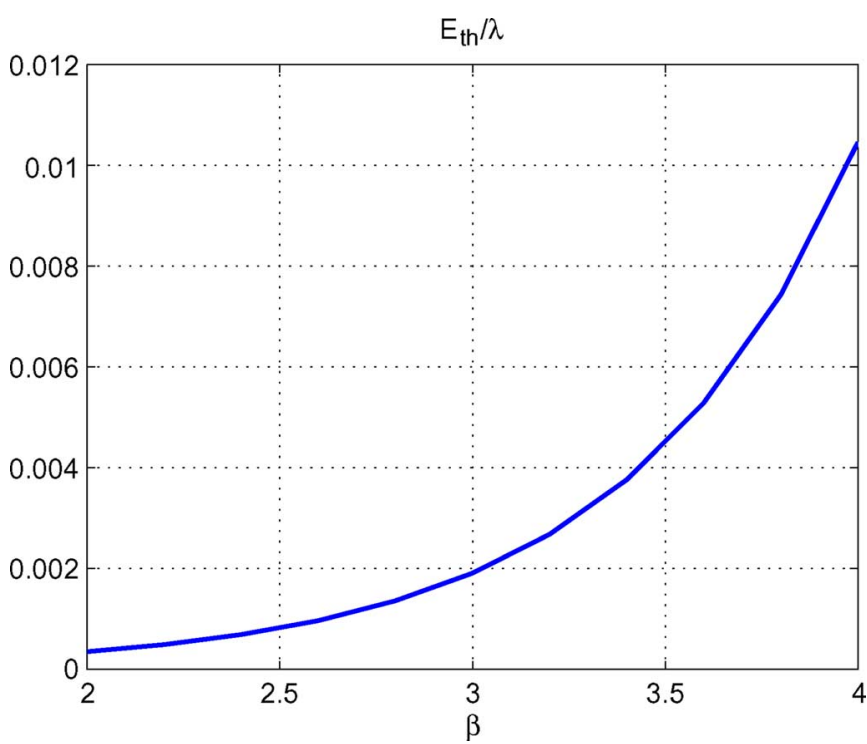

Fig. 6. The computed results for $E_{\mathrm{th}} / \lambda$ with respect to $\beta$.
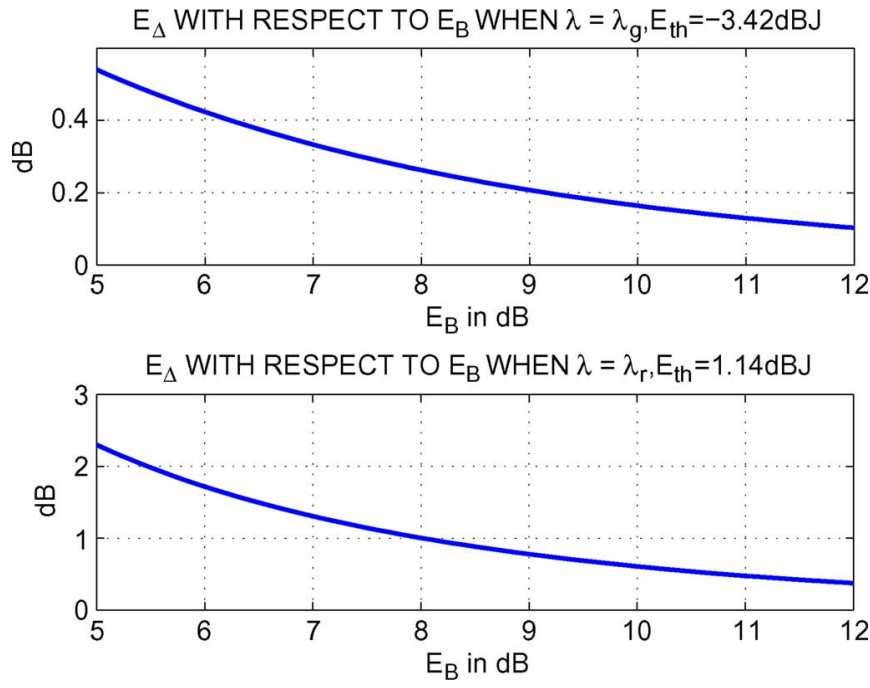

Fig. 7. The computed results for $E_{\triangle}$ with respect to $E_{\mathrm{B}}$ when $\lambda=\lambda_{\mathrm{g}}$ and $\lambda=\lambda_{\mathrm{r}}$, respectively.

the uniform sampling, the number of constraints and computation time is reduced by about $17 \%$ as shown in Fig. 5. Hence, we replace $S$ by $G$ produced with the nonuniform sampling using $\delta=1 \%$ for the following experiments.

In order to show the effect of $\beta$ on $E_{\mathrm{th}}$, we have evaluated $E_{\text {th }} / \lambda$ when $\beta$ varies from 2 to 4 , and the results are shown in Fig. 6. It is shown that $E_{\mathrm{th}} / \lambda$ increases with $\beta$. This means for a fixed $\lambda, E_{\text {th }}$ will increase when signal energy decays faster with respect to distance.

When $E_{\mathrm{B}}$ increases from 5 to $12 \mathrm{dBJ}$, we have computed $E_{\triangle}$ when $\lambda=\lambda_{\mathrm{g}}$ and $\lambda=\lambda_{\mathrm{r}}$, respectively. The results are shown in Fig. 7. It is shown that as $E_{\mathrm{B}}$ increases, $E_{\triangle}$ reduces. When $\lambda=\lambda_{\mathrm{r}}, E_{\triangle}$ is significantly higher than when $\lambda=\lambda_{\mathrm{g}}$. In the following experiments, we choose $E_{\mathrm{B}}=10 \mathrm{dBJ}$, which corresponds to $E_{\triangle}=0.16$ and $0.60 \mathrm{dBJ}$, or equivalently $E_{\mathrm{s}}^{*}=$ -4.15 and $1.75 \mathrm{dBJ}$, when $\lambda=\lambda_{\mathrm{g}}$ and $\lambda=\lambda_{\mathrm{r}}$, respectively. 


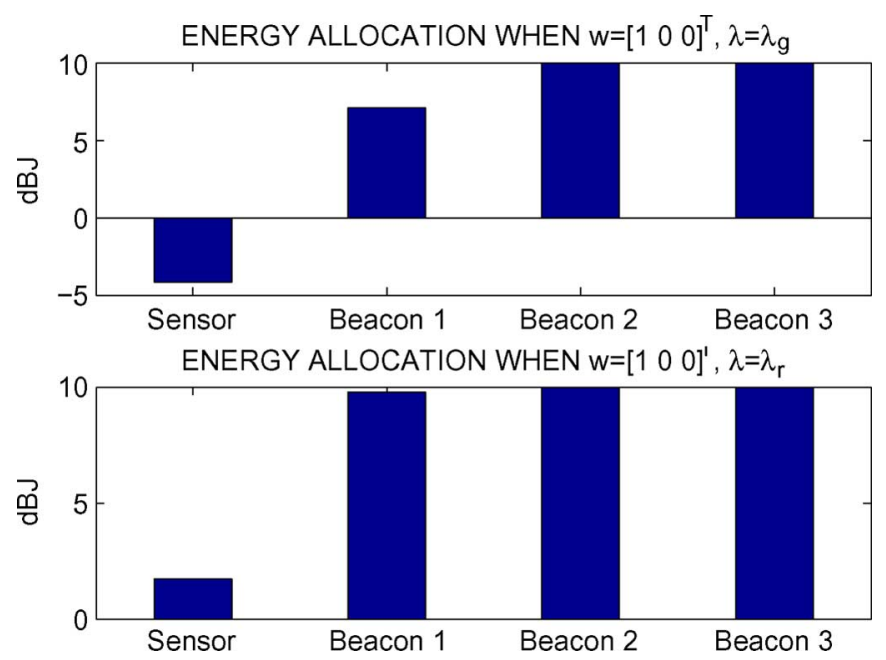

Fig. 8. The computed energy allocation to $E_{\mathrm{s}}$ and $E_{m}$ when $\mathbf{w}=[1,0,0]^{T}$.

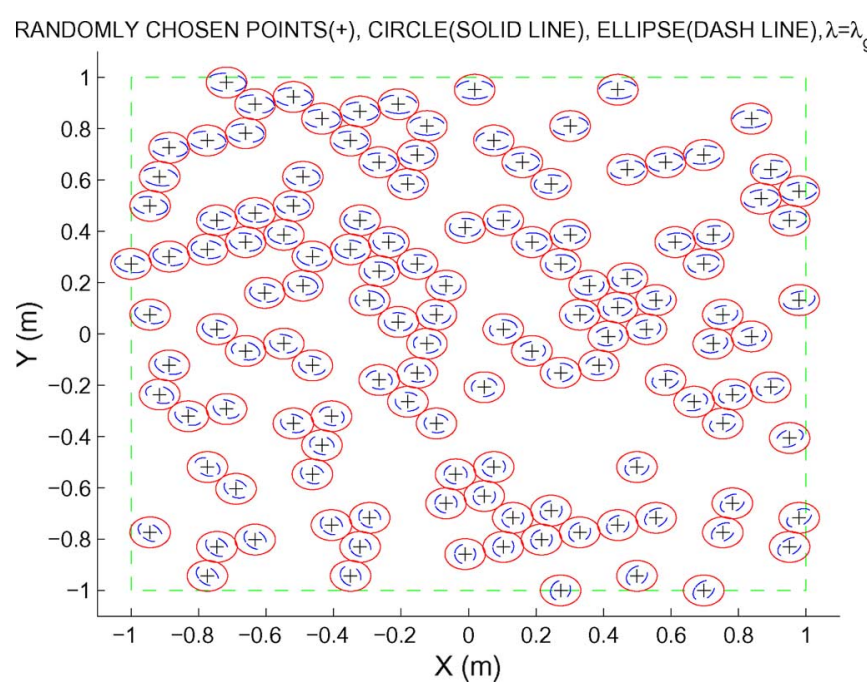

Fig. 9. Error ellipses $\Phi_{e}$ and circles $\Phi_{c}$ for randomly chosen points.

To find a better beacon energy allocation than $E_{m}^{*}=10 \mathrm{dBJ}$, we construct a solution to (14) by (16) using the $\mathrm{x}$ that solves (19). Let's say we want to minimize $E_{1}$ subject to the RSP constraint, so we prescribe the weighting vector as $\mathbf{w}=[1,0,0]^{T}$. The associated problem (19) was solved with Sedumi v1.1, and the $E_{m}^{*}$ 's constructed with the produced $\mathbf{x}^{*}$ are shown in Fig. 8 when $\lambda=\lambda_{\mathrm{g}}$ and $\lambda=\lambda_{\mathrm{r}}$, respectively. The consumed CPU computation time is 11.06 and $11.67 \mathrm{~s}$, respectively. It is shown that the computed $E_{1}^{*}$ is 7.11 and $9.79 \mathrm{dBJ}$, respectively, while $E_{2}^{*}$ and $E_{3}^{*}$ are always $10 \mathrm{dBJ}$.

To show the effectiveness of the energy allocation $E_{\mathrm{s}}^{*}=$ $-4.15 \mathrm{dBJ}, E_{1}^{*}=7.11 \mathrm{dBJ}, E_{2}^{*}=10 \mathrm{dBJ}$, and $E_{3}^{*}=10 \mathrm{dBJ}$ when $\lambda=\lambda_{\mathrm{g}}$, we randomly select a set of points within $S$. We plot the error ellipse $\Phi_{e}=\left\{\mathbf{e}: \mathbf{e}^{T} \mathbf{F}(\mathbf{x}, \mathbf{u}) \mathbf{e} \leq-2 \ln \left(1-P_{e}\right)\right\}$ and the circle $\Phi_{c}=\left\{\mathbf{e}:\|\mathbf{e}\| \leq R_{e}\right\}$ for each point, using the given energy allocation. It is shown in Fig. 9 that each ellipse is enclosed by the associated circle, which indicates that the RSP constraint is indeed satisfied for those randomly chosen points. When $\lambda$ changes from $\lambda_{\mathrm{g}}$ to $\lambda_{\mathrm{r}}$, the energy allocation to $E_{s}^{*}$ should be increased from -4.15 to $1.75 \mathrm{dBJ}$, and that to
$E_{1}^{*}$ should be increased from 7.11 to $9.79 \mathrm{dBJ}$. These energy increases correspond to the extra sensor and beacon energy required to compensate for the unavailable knowledge about the distribution of $\hat{\mathbf{u}}$, when the energy of beacon 1 is used most efficiently.

\section{CONCLUSION}

We have addressed ranging energy optimization for an unsynchronized positioning system, which features RSP in the sense that a specific accuracy requirement is fulfilled within a prescribed service area. We assume a LOS channel exists between the sensor and each beacon. The positioning is implemented by TOA-based TWR between a sensor and beacons, followed by a location estimation at a CPU. To establish a dependency between positioning accuracy and ranging energy, we assume the adopted TOA and location estimators are unbiased and attain the associated CRB. The accuracy requirement has the same form as that defined by the FCC, and we have presented two constraints with LMI form for RSP. Under these constraints, ranging energy optimization problems, as well as a practical algorithm based on SDP have been proposed. We have illustrated the effectiveness of the algorithm by numerical experiments. Although only a 2D system is considered here, the proposed methods can be easily extended to a 3D RSP system. Besides, these methods are not just for UWB based positioning systems, but can be generally applied to other systems as well, as long as the system models and assumptions considered here hold for those systems.

\section{APPENDIX A \\ DERIVATION OF THE CRB FOR ESTIMATING $T_{m \rightarrow \mathrm{s}}$}

Let's consider the estimation of $T_{m \rightarrow s}$ at the sensor. The received signal can be expressed as $r(t)=a_{m} s_{m}\left(t-T_{m \rightarrow \mathrm{s}}\right)+$ $n(t)$, where $n(t)$ is the AWGN with PSD $N_{\mathrm{S}} / 2$. Let's stack all unknown parameters into a vector $\boldsymbol{\theta}=\left[T_{m \rightarrow \mathrm{s}}, a_{m}\right]^{T}$. The $\log$-likelihood function of $\boldsymbol{\theta}$ can be expressed as [10]

$$
\Lambda(\boldsymbol{\theta})=C-\frac{1}{N_{\mathrm{s}}} \int_{-\infty}^{+\infty}\left|r(t)-a_{m} s_{m}\left(t-T_{m \rightarrow \mathrm{s}}\right)\right|^{2} d t
$$

where $C$ keeps unchanged when $\boldsymbol{\theta}$ changes.

The Fisher information matrix (FIM) is $\mathbf{F}_{\boldsymbol{\theta}}=$ $-\mathbb{E}\left(\nabla_{\boldsymbol{\theta}}^{2}(\Lambda(\boldsymbol{\theta}))\right)$, where $\mathbb{E}(\cdot)$ represents the ensemble average operator, and $\nabla_{\boldsymbol{\theta}}^{2}(\Lambda(\boldsymbol{\theta}))$ is the Hessian matrix of $\Lambda(\boldsymbol{\theta})$ with respect to $\boldsymbol{\theta}$. Under the assumptions made in Section II, $\mathbf{F}_{\boldsymbol{\theta}}$ can be reduced to

$$
\mathbf{F}_{\boldsymbol{\theta}}=\left(\begin{array}{cc}
\frac{a_{m}^{2} E_{m}^{\prime}}{N_{\mathrm{s}} / 2} & 0 \\
0 & \frac{E_{m}}{N_{\mathrm{s}} / 2}
\end{array}\right)
$$

where $E_{m}^{\prime}$ is the energy of the differential of $s_{m}(t)$, and thus $E_{m}^{\prime}=\omega_{\mathrm{b}}^{2} E_{m}$. The CRB can then be evaluated as

$$
\mathbf{C}_{\boldsymbol{\theta}}=\left(\mathbf{F}_{\boldsymbol{\theta}}\right)^{-1}=\left(\begin{array}{cc}
\frac{N_{\mathrm{s}} / 2}{a_{m}^{2} \omega_{\mathrm{b}}^{2} E_{m}} & 0 \\
0 & \frac{N_{\mathrm{s}} / 2}{E_{m}}
\end{array}\right) .
$$

Since we assume the above CRB is achieved with an unbiased Gaussian distribution, $\hat{T}_{m \rightarrow \mathrm{s}}$ is distributed as $\mathcal{N}\left(T_{m \rightarrow \mathrm{s}}, N_{\mathrm{s}} /\left(2 a_{m}^{2} \omega_{\mathrm{b}}^{2} E_{m}\right)\right)$. 


\section{APPENDIX B}

\section{DERIVATION OF THE CRB FOR THE LOCATION ESTIMATOR}

Let's consider the estimation of $\mathbf{u}$ using $\left\{\hat{d}_{m, \mathbf{u}}\right\}_{m=1}^{M}$. Note that $\left\{a_{m}\right\}_{m=1}^{M}$ play the role of nuisance parameters. The $\log$-likelihood function of the unknown parameter vector $\boldsymbol{\psi}=\left[\mathbf{u}, a_{1}, \cdots, a_{M}\right]^{T}$ can be expressed as

$$
\Lambda(\boldsymbol{\psi})=\sum_{m=1}^{M=1}\left(-\frac{1}{2} \ln \left(2 \pi \kappa_{m}^{2}\right)-\frac{\left|\hat{d}_{m, \mathbf{u}}-d_{m, \mathbf{u}}\right|^{2}}{2 \kappa_{m}^{2}}\right) .
$$

The Fisher information matrix (FIM) $\mathbf{F}_{\psi}$ can be evaluated as $\mathbf{F}_{\boldsymbol{\psi}}=-\mathbb{E}\left(\nabla_{\boldsymbol{\psi}}^{2}(\Lambda(\boldsymbol{\psi}))\right)$, where $\nabla_{\boldsymbol{\psi}}^{2}(\Lambda(\boldsymbol{\psi}))$ is the Hessian matrix of $\Lambda(\psi)$ with respect to $\psi$. By some arrangements, we can show that the following equalities hold $(m=1, \cdots, M)$ :

$$
\begin{aligned}
& \mathbb{E}\left(\frac{\partial^{2} \Lambda(\boldsymbol{\psi})}{\partial x_{\mathrm{s}} \partial a_{m}}\right)=\mathbb{E}\left(\frac{\partial^{2} \Lambda(\boldsymbol{\psi})}{\partial a_{m} \partial x_{\mathrm{s}}}\right)=0 \\
& \mathbb{E}\left(\frac{\partial^{2} \Lambda(\boldsymbol{\psi})}{\partial y_{\mathrm{s}} \partial a_{m}}\right)=\mathbb{E}\left(\frac{\partial^{2} \Lambda(\boldsymbol{\psi})}{\partial a_{m} \partial y_{\mathrm{s}}}\right)=0 .
\end{aligned}
$$

Using the above equalities, $\mathbf{F}_{\psi}$ can be reduced to

$$
\mathbf{F}_{\boldsymbol{\psi}}=\left(\begin{array}{c|c}
-\mathbb{E}\left(\nabla_{\mathbf{u}}^{2}(\Lambda(\boldsymbol{\psi}))\right) & \mathbf{0}_{M, 2}^{T} \\
\hline \mathbf{0}_{M, 2} & \mathbf{B}_{M, M}
\end{array}\right)
$$

where $\nabla_{\mathbf{u}}^{2}(\Lambda(\boldsymbol{\psi}))$ is the Hessian matrix of $\Lambda(\boldsymbol{\psi})$ with respect to $\mathbf{u}, \mathbf{0}_{M, 2}$ is an all-zero $M \times 2$ matrix, and $\mathbf{B}_{M \times M}$ is the submatrix at the right-bottom corner. It is shown in [45] that

$$
-\mathbb{E}\left(\nabla_{\mathbf{u}}^{2}(\Lambda(\boldsymbol{\psi}))\right)=\mathbb{E}\left(\nabla_{\boldsymbol{\psi}} \Lambda(\boldsymbol{\psi}) \cdot\left(\nabla_{\boldsymbol{\psi}} \Lambda(\boldsymbol{\psi})\right)^{T}\right)
$$

where $\nabla_{\boldsymbol{\psi}} \Lambda(\boldsymbol{\psi})$ is the gradient vector of $\Lambda(\boldsymbol{\psi})$ with respect to $\boldsymbol{\psi}$

$$
\nabla_{\boldsymbol{\psi}} \Lambda(\boldsymbol{\psi})=\sum_{m=1}^{M} \frac{\hat{d}_{m, \mathbf{u}}-d_{m, \mathbf{u}}}{\kappa_{m}^{2}} \frac{\mathbf{u}-\mathbf{p}_{m}}{d_{m, \mathbf{u}}} .
$$

Inserting (28) into (27), we can show that

$$
-\mathbb{E}\left(\nabla_{\mathbf{u}}^{2}(\Lambda(\boldsymbol{\psi}))\right)=\sum_{m=1}^{M} \frac{\left(\mathbf{u}-\mathbf{p}_{m}\right)\left(\mathbf{u}-\mathbf{p}_{m}\right)^{T}}{\kappa_{m}^{2}\left(d_{m, \mathbf{u}}\right)^{2}} .
$$

The CRB for the location estimator is actually the $2 \times 2$ submatrix at the upper-left corner of $\mathbf{F}_{\boldsymbol{\psi}}^{-1}$. By some rearrangement, $\mathbf{C}_{\mathbf{u}}^{-1}$ can be evaluated according to (8).

\section{ACKNOWLEDGMENT}

The authors would like to thank D. Neirynck, F. Shu, and G. Dolmans at Holst Centre for their suggestions. The authors would also thank the anonymous reviewers for their valuable comments.

\section{REFERENCES}

[1] I. F. Akyildiz and W. Su et al., "Wireless sensor networks: A survey," Comput. Netw., vol. 38, no. 4, pp. 393-422, Mar. 2002.

[2] M. G. Rabbat and R. D. Nowak, "Decentralized source localization and tracking [wireless sensor networks]," in Proc. ICASSP, May 2004.

[3] J. Heidemann, F. Silva, and C. Intanagonwiwat et al., "Building efficient wireless sensor networks with low-level naming," ACM SIGOPS Operat. Syst. Rev., vol. 35, no. 5, pp. 146-159, Dec. 2001.
[4] J. N. AI-Karaki and A. E. Kamal, "Routing techniques in wireless sensor networks: A survey," IEEE Wireless Commun., vol. 11, no. 6, pp. 6-28, Dec. 2004

[5] Y.Zhang, W. Liu, W. Lou, and Y. Fang, "Location-based compromisetolerant security mechanisms for wireless sensor networks," IEEE J. Sel. Areas Commun., vol. 24, no. 2, pp. 247-260, Feb. 2006.

[6] FCCRM-8143, "Revision of the Commissions Rules to Insure Compatibility With Enhanced 911 Emergency Calling Systems," Fed. Commun. Comm., Tech. Rep., 1996.

[7] S. Gezici, Z. Tian, and G. B. Giannakis et al., "Localization via ultrawideband radios," IEEE Signal Process. Mag., vol. 22, no. 4, pp. 70-84, Jul. 2005.

[8] F. Gustafsson and F. Gunnarsson, "Mobile positioning using wireless networks," IEEE Signal Process. Mag., vol. 22, no. 4, pp. 41-53, Jul. 2005.

[9] Z. Sahinoglu and S. Gezici, "Ranging in the IEEE 802.15.4a standard," in Proc. Wireless Microw. Technol. Conf., Dec. 2006.

[10] V. Lottici, A. D. Andrea, and U. Mengali, "Channel estimation for ultra-wideband communications," IEEE J. Sel. Areas Commun., vol. 20, no. 12, pp. 1638-1645, Dec. 2002.

[11] J. Y. Lee and R. A. Scholtz, "Ranging in a dense multipath environment using an UWB radio link," IEEE J. Sel. Areas Commun., vol. 20, no. 9, pp. 1677-1683, Dec. 2002.

[12] X. Li and K. Pahlavan, "Super-resolution TOA estimation with diversity for indoor geolocation," IEEE Trans. Wireless Commun., vol. 3, no. 1, pp. 224-234, Jan. 2004.

[13] I. Guvenc and Z. Sahinoglu, "Threshold-based TOA estimation for impulse radio UWB systems," in Proc. ICUWB, Sep. 2005, pp. 420-425.

[14] Z. Tian and L. Wu, "Timing acquisition with noisy template for ultra-wideband communications in dense multipath," EURASIP $J$. Appl. Signal Process., vol. 2005, no. 3, pp. 439-454.

[15] L. Yang and G. B. Giannakis, "Timing ultra-wideband signals with dirty templates," IEEE Trans. Commun., vol. 53, no. 11, pp. 1952-1963, Nov. 2005.

[16] C. Falsi, D. Dardari, and L. Mucchi et al., "Time of arrival estimation for UWB localizers in realistic environments," EURASIP J. Appl. Signal Process., vol. 2006, pp. 1-13.

[17] S. Gezici, Z. Sahinoglu, and A. F. Molisch et al., "Two-step time of arrival estimation for pulse-based ultra-wideband systems," EURASIP J. Appl. Signal Process., vol. 2008, pp. 1-11.

[18] L. Huang and C. C. Ko, "Performance of maximum-likelihood channel estimator for UWB communications," IEEE Commun. Lett., vol. 8, no. 6, pp. 356-358, Jun. 2004.

[19] A. L. Deleuze, C. J. L. Martret, and P. Ciblat et al., "Cramér-Rao bound for channel parameters in ultra-wide band based system," in Proc. SPAWC 2004, Jul. 2004, pp. 140-144.

[20] J. Zhang, R. A. Kennedy, and T. D. Abhayapala, "Cramér-Rao lower bounds for the synchronization of UWB signals," EURASIP J. Appl. Signal Process., vol. 3, pp. 426-438, 2005.

[21] T. Kaiser and B. T. Sieskul, "Cramér-Rao bound for TOA estimations in UWB positioning systems," in Proc. ICUWB, Sep. 2005, pp. 408-413.

[22] N. Bulusu, J. Heidemann, and D. Estrin, "GPS-less low-cost outdoor localization for very small devices," Pers. Commun., vol. 7, no. 5, pp. 28-34, Oct. 2000.

[23] R. L. Moses, D. Krishnamurthy, and R. M. Patterson, "A self-localization method for wireless sensor networks," EURASIP J. Appl. Signal Process., vol. 2003, no. 4, pp. 348-358.

[24] Y. T. Chan, H. Y. C. Hang, and P. C. Ching, "Exact and approximate maximum likelihood localization algorithms," IEEE Trans. Veh. Technol., vol. 55, no. 1, pp. 10-16, Jan. 2006.

[25] K. W. Cheung, H. C. So, and W. K. Ma et al., "Least squares algorithms for time-of-arrival-based mobile location," IEEE Trans. Signal Process., vol. 52, no. 4, pp. 1121-1128, Apr. 2004.

[26] K. W. Cheung, H. C. So, and W. K. Ma et al., "A constrained least squares approach to mobile positioning algorithms and optimality," EURASIP J. Appl. Signal Process., vol. 2006, pp. 1-23.

[27] K. W. Cheung, W. K. Ma, and H. C. So, "Accurate approximation algorithm forTOAa-based maximum likelihood mobile location using semidefinite programming," in Proc. ICASSP 2004, 2004, pp. 145-148.

[28] C. Meng, Z. Ding, and S. Dasgupta, "A semidefinite programming approach to source localization in wireless sensor networks," IEEE Signal Process. Lett., vol. 15, pp. 253-256, 2008.

[29] K. W. Cheung and H. C. So, "A multidimensional scaling framework for mobile location using time-of-arrival measurements," IEEE Trans. Signal Process., vol. 53, no. 2, pp. 460-470, Feb. 2005. 
[30] H. C. So and F. K. W. Chan, "A generalized subspace approach for mobile positioning with time-of-arrival measurements," IEEE Trans. Signal Process., vol. 55, no. 10, pp. 5103-5107, Oct. 2007.

[31] H. Wei, W. Wang, and Z. Chen et al., "A novel weighted multidimensional scaling analysis for time-of-arrival-based mobile location," IEEE Trans. Signal Process., vol. 55, no. 7, pp. 3018-3022, Jul. 2008.

[32] K. Langendoen and N. Reijers, "Distributed localization in wireless sensor networks: A quantitative comparison," Comput. Netw., vol. 43, pp. 499-518, 2003.

[33] A. T. Ihler, J. W. Fisher, III, and R. L. Moses et al., "Nonparametric belief propagation for self-localization of sensor networks," IEEE $J$. Sel. Areas Commun., vol. 23, no. 4, pp. 809-819, Apr. 2005.

[34] P. Biswas, T. C. Liang, and T. C. Wang et al., "Semidefinite programming based algorithms for sensor network localization," ACM Trans. Sens. Netw., vol. 2, no. 2, pp. 188-220, May 2006.

[35] S. Srirangarajan, A. H. Tewfik, and Z. Q. Luo, "Distributed sensor network localization with inaccurate anchor positions and noisy distance information," in Proc. ICASSP 2007.

[36] J. A. Costa, N. Patwari, and A. O. Hero, III et al., "Distributed weighted-multidimensional scaling for node localization in sensor networks," ACM Trans. Sens. Netw., vol. 2, no. 1, pp. 39-64, Feb. 2006.

[37] G. Latsoudas and N. D. Sidiropoulos, "A fast and effective multidimensional scaling approach for node localization in wireless sensor networks," IEEE Trans. Signal Process., vol. 55, no. 10, pp. 5121-5127, Oct. 2007.

[38] A. Catovic and Z. Sahinoglu, "The Cramér-Rao bounds of hybrid TOA/RSS and TDOA/RSS location estimation schemes," IEEE Commun. Lett., vol. 8, no. 10, pp. 626-628, Oct. 2004.

[39] J. Y. Huang and Q. Wan, "Comments on the Cramér-Rao bounds of hybrid TOA/RSS and TDOA/RSS location estimation schemes," IEEE Commun. Lett., vol. 11, no. 11, pp. 848-849, Nov. 2007.

[40] D. B. Jourdan, D. Dardari, and M. Z. Win, "Position error bound for UWB localization in dense cluttered environments," IEEE Trans. Aerosp. Electron. Syst., vol. 44, no. 2, pp. 613-628, Apr. 2008.

[41] N. Patwari, A. O. Hero, III, and M. Perkins et al., "Relative location estimation in wireless sensor networks," IEEE Trans. Signal Process., vol. 51, no. 8, pp. 2137-2148, Aug. 2003.

[42] E. G. Larsson, "Cramér-Rao bound analysis of distributed positioning in sensor networks," IEEE Signal Process. Lett., vol. 2004, no. 3, pp. 334-337, Mar. 2004.

[43] A. Savvides, W. L. Garber, and R. L. Moses et al., "An analysis of error inducing parameters in multihop sensor node localization," IEEE Trans. Mobile Comput., vol. 4, no. 6, pp. 567-577, Nov. 2005.

[44] C. Chang and A. Sahai, "Cramér-Rao-type bounds for localization," EURASIP J. Appl. Signal Process., vol. 2006, pp. 1-13.

[45] S. M. Kay, Fundamentals of Statistical Signal Processing-Estimation Theory. Englewood Cliffs, NJ: Prentice-Hall, 1993.

[46] D. J. Torrieri, "Statistical theory of passive location systems," IEEE Trans. Aerosp. Electron. Syst., vol. 20, no. 2, pp. 183-197, Mar. 1984.

[47] B. M. Sadler, L. Huang, and Z. Xu, "Ziv-Zakai time delay estimation bound for ultra-wideband signals," in Proc. ICASSP, Apr. 2007, pp. $549-552$.

[48] Z. Xu and B. M. Sadler, "Time delay estimation bounds in concolutive random channels," IEEE J. Sel. Topics Signal Process., vol. 1, no. 3, pp. 418-430, Oct. 2007.

[49] S. Boyd and L. Vandenberghe, Convex Optimization. Cambridge, U.K.: Cambridge Univ. Press, 2004.

[50] T. M. Cover and J. A. Thomas, Elements of Information Theory, 2nd ed. New York: Wiley, 2006.

[51] R. Horn and C. R. Johnson, Matrix Analysis. Cambridge, U.K.: Cambridge Univ. Press, 1985.
[52] J. S. Sturm, "Using SeDuMi 1.02 a Matlab toolbox for optimization over symmetric cones," Optimiz., Methods Softw., no. 10-12, pp. 625-653, 1999.

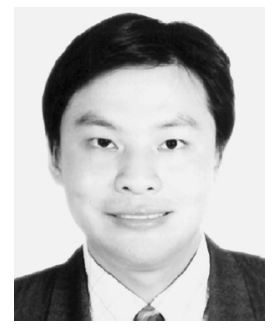

Tao Wang received the B.E. and Do.E. degrees in electronic engineering from Zhejiang University, China, in June 2001 and June 2006, respectively.

Since August 2000 to February 2001, he had an internship on the design and implementation of a microcontrol unit with Motorola Electronics Ltd. Suzhou Branch, China. In October 2004 to March 2005, he was a visiting student with the Institute for Infocomm Research, Singapore, working on ultrawideband antenna design. His current research interests are in the design of statistical signal processing algorithms, as well as optimization of wireless localization and communication systems.

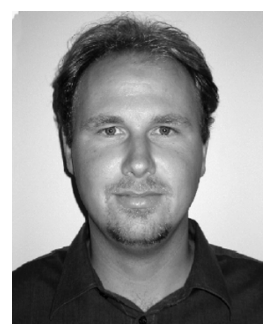

Geert Leus (M'01-SM'05) was born in Leuven, Belgium, in 1973. He received the Electrical Engineering and Ph.D. degrees in applied sciences from the Katholieke Universiteit Leuven, in June 1996 and May 2000, respectively.

$\mathrm{He}$ has been a Research Assistant and a Postdoctoral Fellow of the Fund for Scientific Research-Flanders, Belgium, from October 1996 until September 2003. During that period, he was with the Electrical Engineering Department, Katholieke Universiteit Leuven. Currently, he is an Associate Professor with the Faculty of Electrical Engineering, Mathematics and Computer Science, Delft University of Technology, The Netherlands. During summer 1998, he visited Stanford University, Stanford, CA, and from March 2001 until May 2002, he was a Visiting Researcher and Lecturer with the University of Minnesota, Minneapolis. His research interests are in the area of signal processing for communications.

Dr. Leus received a 2002 IEEE Signal Processing Society Young Author Best Paper Award and a 2005 IEEE Signal Processing Society Best Paper Award. $\mathrm{He}$ is the Chair of the IEEE Signal Processing for Communications Technical Committee, and an Associate Editor for the IEEE TRANSACTIONS ON SIGNAL PROCESSING and the EURASIP Journal on Applied Signal Processing. In the past, he has served on the Editorial Board of the IEEE Signal PROCESSING LETTERS and the IEEE TRANSACTIONS ON WIRELESS COMMUNICATIONS.

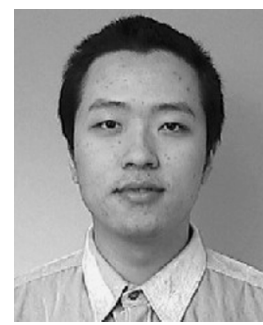

Li Huang (S'04-M'08) received the B.Eng. degree in electronics and information engineering in 2002 from Huazhong University of Science Technology (HUST), Wuhan, China, and the Ph.D. degree from both the National University of Singapore (NUS) and the Technical University Eindhoven (TU/e), The Netherlands, in 2008

Since 2006, he has been with IMEC-NL, Holst Centre, Eindhoven, working on system-level design for ultralow power wireless systems. His main research interests include statistical signal processing, channel modeling, medium access control (MAC) design, wireless communications, and magnetic and optical recording. 